\title{
Decidability of Weak Simulation on One-counter Nets*
}

\author{
Piotr Hofman ${ }^{1}$, Richard Mayr ${ }^{2}$, and Patrick Totzke ${ }^{2}$ \\ ${ }^{1}$ University of Warsaw, Poland \\ ${ }^{2}$ University of Edinburgh, UK
}

\begin{abstract}
One-counter nets $(\mathrm{OCN})$ are Petri nets with exactly one unbounded place. They are equivalent to a subclass of one-counter automata with only a weak test for zero.

We show that weak simulation preorder is decidable for OCN and that weak simulation approximants do not converge at level $\omega$, but only at $\omega^{2}$. In contrast, other semantic relations like weak bisimulation are undecidable for OCN [17], and so are weak (and strong) trace inclusion (Sec. 7).
\end{abstract}

\section{Introduction}

The model. One-counter automata (OCA) are Minsky counter automata with only one counter, and they can also be seen as a subclass of pushdown automata with just one stack symbol (plus a bottom symbol). One-counter nets (OCN) are Petri nets with exactly one unbounded place, and they correspond to a subclass of OCA where the counter cannot be fully tested for zero (i.e., transitions enabled at counter value zero are also enabled at nonzero counter values). OCN are arguably the simplest model of discrete infinite-state systems, except for those that do not have a global finite control, e.g., (commutative) context-free grammars.

The state of the art. Verification problems for OCA and OCN have been extensively studied, particularly model checking problems with various temporal logics and semantic preorder/equivalence checking w.r.t. given notions of behavior. $\mu$-calculus model checking $[20,8]$ and CTL model checking [7] are PSPACE-complete for OCA/OCN, while EF model checking is $P^{N P}$-complete [8]. There are many notions of semantic equivalences [6], but the most common ones are the following (ordered from finer to coarser): bisimulation, simulation and trace equivalence. Each of these have their standard (called strong) variant, and a weak variant that abstracts from arbitrarily long sequences of internal actions.

Strong bisimulation for OCA/OCN is PSPACE-complete [3]. However, OCA and OCN differ w.r.t. strong simulation. While strong simulation is decidable for OCN $[1,12,11]$, strong simulation and trace inclusion are undecidable for OCA [13]. ((Bi)simulation checking is also undecidable for models with more than one unbounded counter/place [9].)

The situation changes when one considers weak semantic equivalences/preorders that abstract from internal actions. One can define upper-approximations of (bi)simulation up-to $k$ by considering only $k$ rounds in the (bi)simulation game. For strong (bi)simulation, these $k$ approximants converge to (bi)simulation at level $k=\omega$, provided that the systems are finitely

\footnotetext{
${ }^{*}$ Technical Report EDI-INF-RR-1415 of the School of Informatics at the University of Edinburgh, UK. (http://www.inf.ed.ac.uk/publications/report/). Extended version of material presented at LICS 2013. Made available at arXiv.org - Non-exclusive license to distribute (http://arxiv.org/licenses/nonexclusive-distrib/1.0/).
} 
branching. This is not the case for weak (bi)simulation. Here the approximants are guaranteed to converge only at higher ordinals, due to the implicit infinite branching capability introduced by the abstraction. This is why it is so hard to prove semi-decidability of weak non-(bi)simulation for many classes of infinite-state transition systems.

For OCA/OCN it was shown that weak bisimulation is undecidable [17]. Moreover, weak (and strong) simulation and trace inclusion are undecidable for OCA [13, 22]. However, it was an open question whether weak simulation is decidable for OCN. Moreover, the decidability of strong and weak trace inclusion was open for OCN [4].

Our contribution. We show that weak simulation preorder is decidable for OCN. In fact, the weak simulation relation on OCN is effectively semilinear. Moreover, we show that weak simulation approximants only converge at level $\omega^{2}$ on OCN. The decidability of weak simulation is in contrast to the undecidability of weak bisimulation for OCN [17]. This is surprising, because it goes against a common trend. On almost all other classes of systems, bisimulation problems are computationally easier than the corresponding simulation problems [14].

On the other hand, we show that strong and weak trace inclusion are undecidable even for OCN.

Finally, we study checking strong and weak (bi)simulation and trace inclusion between $\mathrm{OCA} / \mathrm{OCN}$ and finite systems, and close some remaining complexity gaps in this area.

\section{Preliminaries}

One-counter systems. We consider infinite-state labeled transition systems induced by OCA and /OCN, respectively. A labeled transition system is described by a triple $T=(V, A c t, \longrightarrow)$ where $V$ is a (possibly infinite) set of states, Act is a finite set of action labels and $\longrightarrow \subseteq$ $V \times A c t \times V$ is the labeled transition relation. We write $\longrightarrow^{*}$ for the transitive and reflexive closure of $\longrightarrow$ and use the infix notation $s \stackrel{a}{\longrightarrow} s^{\prime}$ for a transition $\left(s, a, s^{\prime}\right) \in \longrightarrow$, in which case we say $T$ makes an $a$-step from $s$ to $s^{\prime}$. Given a finite or infinite sequence of symbols $w \in A c t^{*}$ or $w \in A c t^{\omega}$ resp., we write $|w| \in \mathbb{N} \cup\{\omega\}$ for the length of $w$. If $w$ is finite, we denote its $i$-fold concatenation by $w^{i}$.

Definition 1 (One-Counter Automata / Nets) $A$ one-counter automaton $A=\left(Q, A c t, \delta, \delta_{0}\right)$ is given by a finite set of control-states $Q$, a finite set of action labels Act and transition relations $\delta \subseteq Q \times A c t \times\{-1,0,1\} \times Q$ and $\delta_{0} \subseteq Q \times$ Act $\times\{0,1\} \times Q$. It induces an infinite-state labeled transition system over the stateset $Q \times \mathbb{N}$, whose elements will be written as pm, as follows. $p m \stackrel{a}{\longrightarrow} p^{\prime} m^{\prime}$ iff

1. $\left(p, a, d, p^{\prime}\right) \in \delta$ and $m^{\prime}=m+d \geq 0$ or

2. $\left(p, a, d, p^{\prime}\right) \in \delta_{0}, m=0$ and $m^{\prime}=d$.

Such an automaton is called $a$ one-counter net if $\delta_{0}=\emptyset$, i.e., if the automaton cannot test if the counter is equal to 0.

Weak Simulation. In a weak semantics, one needs to abstract from internal actions. Thus one assumes a dedicated action $\tau \in A c t$ that is used to model internal non-observable steps and defines the weak step relation $\Longrightarrow$ by

$$
\stackrel{\tau}{\Longrightarrow}=\stackrel{\tau}{\longrightarrow} \text {, and for } a \neq \tau, \quad \quad \stackrel{a}{\longrightarrow}=\stackrel{\tau}{\longrightarrow} * \stackrel{a}{\longrightarrow} \cdot \stackrel{\tau}{\longrightarrow} *
$$

Simulation and weak simulation are semantic preorders in van Glabbeeks linear time branching time spectrum [6], which are used to compare the behavior of processes. Their standard co-inductive definition is as follows. A binary relation $R \subseteq V^{2}$ on the states of a labeled 
transition system is a simulation if $s R t$ implies that for all $s \stackrel{a}{\longrightarrow} s^{\prime}$ there is a $t^{\prime}$ such that $t \stackrel{a}{\longrightarrow} t^{\prime}$ and $s^{\prime} R t^{\prime}$. Similarly, $R$ is a weak simulation if $s R t$ implies that for all $s \stackrel{a}{\longrightarrow} t$ there is a $t^{\prime}$ such that $t \stackrel{a}{\Longrightarrow} t^{\prime}$ and $s^{\prime} R t^{\prime}$. (Weak) simulations are closed under union, so there exists a unique maximal simulation $\preceq$, resp. weak simulation $\leqq$, which is a preorder on $V$. A (weak) bisimulation is a symmetric (weak) simulation. The maximal (weak) bisimulation is an equivalence.

Simulation preorder can also be characterized in terms of ordinal approximant relations $\preceq_{\alpha}$, which are inductively defined as follows. $\preceq_{0}=V^{2}$. For successors $\alpha+1$ let $s \preceq_{\alpha+1} t$ iff for all $s \stackrel{a}{\longrightarrow} s^{\prime}$ there is a $t \stackrel{a}{\longrightarrow} t^{\prime}$ such that $s^{\prime} \preceq_{\alpha} t^{\prime}$. For limit ordinals $\lambda$ define $\preceq_{\lambda}=\bigcap_{\alpha<\lambda} \preceq_{\alpha}$.

This inductive notion of approximants can be interpreted as an interactive game between two players Spoiler and Duplicator, where the latter tries to stepwise match the moves of the former. A play is a finite or infinite sequence of pairs of transition system states. For a finite play $\left(E_{0}, F_{0}\right),\left(E_{1}, F_{1}\right), \ldots,\left(E_{i}, F_{i}\right)$ the next pair $\left(E_{i+1}, F_{i+1}\right)$ is determined by a round of choices: Spoiler chooses a transition $E_{i} \stackrel{a}{\longrightarrow} E_{i+1}$, then Duplicator responds by choosing an equally labeled transition $F_{i} \stackrel{a}{\longrightarrow} F_{i+1}$. A pair $(E, F)$ of states is directly winning for Spoiler if she can choose a transition $E \stackrel{a}{\longrightarrow} E^{\prime}$ so that her opponent cannot respond, i.e. $\neg \exists F^{\prime} . F \stackrel{a}{\longrightarrow} F^{\prime}$. A play is won by Spoiler if a pair of states is reached that is directly winning for her, otherwise Duplicator wins the play. A strategy is a set of rules that tells the player which valid move to choose. A player plays according to a strategy if all her moves obey the rules of the strategy. A strategy is winning from $(E, F)$ if every play that starts in $(E, F)$ and which is played according to that strategy is winning.

Proposition 2 For any two states $(E, F)$ of a transition system $T$, Duplicator has a winning strategy in $(E, F)$ in the simulation game iff $E \preceq_{\alpha} F$ for all ordinals $\alpha$ iff $E \preceq F$.

Weak simulation approximants $\leqq_{\alpha}$ and games are defined analogously but allow Duplicator to make weak steps and characterize $\leqq$.

The Problem. We consider the problem of deciding weak simulation preorder on one-counter nets. An instance is given by a one-counter net $N=(Q, A c t, \delta)$ and configurations $p m$ and $q n$, and the question is whether $p m \leqq q n$ holds. Generally, we want to compute a representation of the semilinear set $W(p, q)=\{(m, n) \mid p m \leqq q n\}$.

\section{Reduction to Strong Simulation on $\omega$-Nets}

First we reduce the weak simulation problem on one-counter nets to a strong simulation problem on a slightly generalized model that we call $\omega$-nets. In $\omega$-nets, there exist dedicated transitions with symbolic effect $\omega$, which allow to arbitrarily increase the counter in a single step. Checking weak simulation between two one-counter nets can be reduced to strong simulation between a one-counter net and an $\omega$-net.

Definition $3(\omega$-Nets) An $\omega$-net $N=(Q$, Act,$\delta)$ is given by a finite set of control-states $Q, a$ finite set of actions Act and transitions $\delta \subseteq Q \times A c t \times\{-1,0,1, \omega\} \times Q$. It induces a transition system over the stateset $Q \times \mathbb{N}$ that allows a step $p m \stackrel{a}{\longrightarrow} p^{\prime} m^{\prime}$ if either $\left(p, a, d, p^{\prime}\right) \in \delta$ and $m^{\prime}=m+d \in \mathbb{N}$ or if $\left(p, a, \omega, p^{\prime}\right) \in \delta$ and $m^{\prime}>m$.

Every one-counter net is a $\omega$-net without $\omega$-transitions. Unlike one-counter nets, $\omega$-nets can yield infinitely branching transition systems, since each $\omega$-transition $\left(p, a, \omega, p^{\prime}\right)$ introduces steps $p m \stackrel{a}{\longrightarrow} p^{\prime} m^{\prime}$ for any two naturals $m^{\prime}>m$.

It is easily verified that $\omega$-net (and hence also one-counter net) processes satisfy the following monotonicity property. 
Proposition 4 (Monotonicity of $\preceq$ ) $p m \stackrel{a}{\longrightarrow} p^{\prime} m^{\prime}$ implies $p(m+d) \stackrel{a}{\longrightarrow} p^{\prime}\left(m^{\prime}+d\right)$ for all $d \in$ $\mathbb{N}$. Moreover, $p m \preceq q n$ implies $p m^{\prime} \preceq q n^{\prime}$ for $m^{\prime} \leq m, n^{\prime} \geq n$.

The following theorem justifies our focus on strong simulation games where Duplicator plays on an $\omega$-net process.

Theorem 5 Checking weak simulation between two one-counter net processes can be reduced to checking strong simulation between a one-counter net process and an $\omega$-net process. Formally, for two one-counter nets $M$ and $N$ with states $Q_{M}$ and $Q_{N}$ resp., one can effectively construct a OCN $M^{\prime}$ with states $Q_{M^{\prime}} \supseteq Q_{M}$ and a $\omega$-net $N^{\prime}$ with states $Q_{N^{\prime}} \supseteq Q_{N}$ such that for each pair $p, q \in Q_{M} \times Q_{N}$ of original control states and any ordinal $\alpha$ the following hold.

1. $p m \leqq q n$ w.r.t. $M, N$ iff $p m \preceq q n$ w.r.t. $M^{\prime}, N^{\prime}$.

2. If $p m \leqq_{\alpha} q n$ w.r.t. $M, N$ then $p m \preceq_{\alpha}$ qn w.r.t. $M^{\prime}, N^{\prime}$.

Proof (Sketch.) The idea of the proof is to look for counter-increasing cyclic paths via $\tau$-labeled transitions in the control graph and to introduce $\omega$-transitions accordingly. For any path that reads a single visible action and visits a 'generator' state that is part of a silent cycle with positive effect, we add an $\omega$-transition. For all of the finitely many non-cyclic paths that read a single visible action we introduce direct transitions. A full proof is given in Appendix A.

\section{Approximants}

We generalize the notion of $\preceq_{\alpha}$ simulation approximants in the case of simulation between onecounter and $\omega$-net processes. This yields approximants that converge at a finite level for any pair of nets.

First we define approximants $\preceq_{\alpha}^{\beta}$ in two (ordinal) dimensions. From a game-theoretic perspective the subscript $\alpha$ indicates the number of rounds Duplicator can survive and the superscript $\beta$ denotes the number of $\omega$-steps Spoiler needs to allow. E.g., $p m \preceq_{5}^{2} q n$ if Duplicator can guarantee that no play of the simulation game that contains $<2 \omega$-steps is losing for him in less than 6 rounds. If not stated otherwise we assume that $N=(Q, A c t, \delta)$ is a one-counter net and $N^{\prime}=\left(Q^{\prime}\right.$, Act, $\left.\delta^{\prime}\right)$ is an $\omega$-net.

Definition 6 We define approximants for ordinals $\alpha$ and $\beta$ as follows. Let $\preceq_{\alpha}^{0}=\preceq_{0}^{\beta}=Q \times$ $\mathbb{N} \times Q^{\prime} \times \mathbb{N}$, the full relation. For successor ordinals $\alpha+1, \beta+1$ let pm $\preceq_{\alpha+1}^{\beta+1}$ qn iff for all $p m \stackrel{a}{\longrightarrow} p^{\prime} m^{\prime}$ there is a step $q n \stackrel{a}{\longrightarrow} q^{\prime} n^{\prime}$ s.t. either

1. $\left(q, a, \omega, q^{\prime}\right) \in \delta^{\prime}$ (the step is due to an $\omega$-transition) and $p^{\prime} m^{\prime} \preceq_{\alpha}^{\beta} q^{\prime} n^{\prime}$, or

2. $\left(q, a, \omega, q^{\prime}\right) \notin \delta^{\prime}$, but $\left(q, a,\left(n^{\prime}-n\right), q^{\prime}\right) \in \delta^{\prime}$ (i.e., there is no $\omega$-transition and the step is due to a normal transition) and $p^{\prime} m^{\prime} \preceq_{\alpha}^{\beta+1} q^{\prime} n^{\prime}$.

For limit ordinals $\lambda$ we define $\preceq_{\alpha}^{\lambda}=\bigcap_{\beta<\lambda} \preceq_{\alpha}^{\beta}$ and $\preceq_{\lambda}^{\beta}=\bigcap_{\alpha<\lambda} \preceq_{\alpha}^{\beta}$. Finally,

$$
\preceq^{\beta}=\bigcap_{\alpha \in \text { Ord }} \preceq_{\alpha}^{\beta} \quad \preceq_{\alpha}=\bigcap_{\beta \in O r d} \preceq_{\alpha}^{\beta} .
$$

$\preceq_{\alpha}$ corresponds to the usual notion of simulation approximants and $\preceq^{\beta}$ is a special notion derived from the syntactic peculiarity of $\omega$-transitions present in the game on one-counter vs. $\omega$-nets. 
Example 7 Consider a net that consists of a single a-labeled loop in state $X$ and the $\omega$-net with transitions $Y \stackrel{a, \omega}{\longrightarrow} Z \stackrel{a,-1}{\longrightarrow} Z$ only. We see that for any $m, n \in \mathbb{N}, X m \preceq_{n} Z n \beth_{n+1} X m$. Moreover, $X m \preceq_{\omega} Y n$ but $X m \npreceq_{\omega+1} Y n$ and $X m \preceq^{1} Y n$ but $X m \npreceq_{\omega+1}^{2} Y n$ and thus $X m \npreceq^{2} Y n$.

Definition 8 An approximant game is played in rounds between Spoiler and Duplicator. Game positions are quadruples $(p m, q n, \alpha, \beta)$ where $p m$, qn are configurations of $N$ and $N^{\prime}$ respectively, and $\alpha, \beta$ are ordinals called step- and $\omega$-counter. In each round that starts in $(p m, q n, \alpha, \beta)$ :

- Spoiler chooses ordinals $\alpha^{\prime}<\alpha$ and $\beta^{\prime}<\beta$,

- Spoiler makes a step $p m \stackrel{a}{\longrightarrow} p^{\prime} m^{\prime}$,

- Duplicator responds by making a step $q n \stackrel{a}{\longrightarrow} q^{\prime} n^{\prime}$ using some transition $t$.

If $t$ was an $\omega$-transition the game continues from position $\left(p^{\prime} m^{\prime}, q^{\prime} n^{\prime}, \alpha^{\prime}, \beta^{\prime}\right)$, Otherwise the next round starts at $\left(p^{\prime} m^{\prime}, q^{\prime} n^{\prime}, \alpha^{\prime}, \beta\right)$ (in this case Spoiler's choice of $\beta^{\prime}$ becomes irrelevant). If a player cannot move the other wins and if $\alpha$ or $\beta$ becomes 0 , Duplicator wins.

Lemma 9 If Duplicator wins the approximation game from $(p m, q n, \alpha, \beta)$ then he also wins the game from $\left(p m, q n, \alpha^{\prime}, \beta^{\prime}\right)$ for any $\alpha^{\prime} \leq \alpha$ and $\beta^{\prime} \leq \beta$.

Proof If Duplicator has a winning strategy in the game from $(p m, q n, \alpha, \beta)$ then he can use the same strategy in the game from $\left(p m, q n, \alpha^{\prime}, \beta^{\prime}\right)$ and maintain the invariant that the pair of ordinals in the game configuration is pointwise smaller than the pair in the original game. Thus Duplicator wins from $\left(p m, q n, \alpha^{\prime}, \beta^{\prime}\right)$.

Lemma $10 \mathrm{pm} \preceq_{\alpha}^{\beta}$ qn iff Duplicator has a strategy to win the approximation game that starts in $(p m, q n, \alpha, \beta)$.

Proof We show both directions by well-founded induction on the pairs of ordinals $(\alpha, \beta)$.

For the "only if" direction we assume $p m \preceq_{\alpha}^{\beta} q n$ and show that Duplicator wins the game from $(p m, q n, \alpha, \beta)$. In the base case of $\alpha=0$ or $\beta=0$ Duplicator directly wins by definition. By induction hypothesis we assume that the claim is true for all pairs pointwise smaller than $(\alpha, \beta)$. Spoiler starts a round by picking ordinals $\alpha^{\prime}<\alpha$ and $\beta^{\prime}<\beta$ and moves $p m \stackrel{a}{\longrightarrow} p^{\prime} m^{\prime}$. We distinguish two cases, depending on whether $\beta$ is a limit or successor ordinal.

Case 1: $\beta$ is a successor ordinal. By Lemma 9 we can safely assume that $\beta^{\prime}=\beta-1$. By our assumption $p m \preceq_{\alpha}^{\beta} q n$ and Def. 6, there must be a response $q n \stackrel{a}{\longrightarrow} q^{\prime} n^{\prime}$ that is either due to an $\omega$-transition and then $p^{\prime} m^{\prime} \preceq_{\alpha^{\prime}}^{\beta^{\prime}} q^{\prime} n^{\prime}$ or due to an ordinary transition, in which case we have $p^{\prime} m^{\prime} \preceq_{\alpha^{\prime}}^{\beta^{\prime}+1} q^{\prime} n^{\prime}$. In both cases, we know by the induction hypothesis that Duplicator wins from this next position and thus also from the initial position.

Case 2: $\beta$ is a limit ordinal. By $p m \preceq_{\alpha}^{\beta} q n$ and Def. 6 , we obtain $p m \preceq_{\alpha}^{\gamma} q n$ for all $\gamma<\beta$. If $\alpha$ is a successor ordinal then, by Lemma 9 , we can safely assume that $\alpha^{\prime}=\alpha-1$. Otherwise, if $\alpha$ is a limit ordinal, then, by Def. 6, we have $p m \preceq_{\alpha^{\prime \prime}}^{\gamma} q n$ for all $\alpha^{\prime \prime}<\alpha$ and in particular $p m \preceq_{\alpha^{\prime}+1}^{\gamma} q n$. So in either case we obtain

$$
p m \preceq_{\alpha^{\prime}+1}^{\gamma} q n \text { for all } \gamma<\beta \text {. }
$$

If there is some $\omega$-transition that allows a response $q n \stackrel{a}{\longrightarrow} q^{\prime} n^{\prime}$ that satisfies $p^{\prime} m^{\prime} \preceq_{\alpha^{\prime}}^{\beta^{\prime}} q^{\prime} n^{\prime}$, then Duplicator picks this response and we can use the induction hypothesis to conclude that he wins the game from the next position. Otherwise, if no such $\omega$-transition exists, Equation (2) 
implies that for every $\gamma<\beta$ there is a response to some $q^{\prime} n^{\prime}$ that uses a non- $\omega$-transition $t(\gamma)$ and that satisfies $p^{\prime} m^{\prime} \preceq_{\alpha^{\prime}}^{\gamma} q^{\prime} n^{\prime}$. Since $\beta$ is a limit ordinal, there exist infinitely many $\gamma<\beta$. By the pigeonhole principle, that there must be one transition that occurs as $t(\gamma)$ for infinitely many $\gamma$. Therefore, a response that uses this transition satisfies $p^{\prime} m^{\prime} \preceq_{\alpha^{\prime}}^{\beta} q^{\prime} n^{\prime}$. If Duplicator uses this response, the game continues from position $\left(p^{\prime} m^{\prime}, q^{\prime} n^{\prime}, \alpha^{\prime}, \beta\right)$ and he wins by induction hypothesis.

For the "if" direction we show that $p m \npreceq_{\alpha}^{\beta} q n$ implies that Spoiler has a winning strategy in the approximation game from $(p m, q n, \alpha, \beta)$. In the base case of $\alpha=0$ or $\beta=0$ the implication holds trivially since the premise is false. By induction hypothesis we assume that the implication is true for all pairs pointwise smaller than $(\alpha, \beta)$. Observe that if $\alpha$ or $\beta$ are limit ordinals then (by Def. 6) there are successors $\beta^{\prime} \leq \beta$ and $\alpha^{\prime} \leq \alpha$ s.t. pm $\npreceq_{\alpha^{\prime}}^{\beta^{\prime}} q n$. So without loss of generality we can assume that $\alpha$ and $\beta$ are successors. By the definition of approximants there must be a move $p m \stackrel{a}{\longrightarrow} p^{\prime} m^{\prime}$ s.t.

- for every possible response $q n \stackrel{a}{\longrightarrow} q^{\prime} n^{\prime}$ that uses some $\omega$-transition we have $p^{\prime} m^{\prime} \npreceq_{\alpha-1}^{\beta-1} q^{\prime} n^{\prime}$,

- for every possible response $q n \stackrel{a}{\longrightarrow} q^{\prime} n^{\prime}$ via some normal transition it holds that $p^{\prime} m^{\prime} \npreceq_{\alpha-1}^{\beta}$ $q^{\prime} n^{\prime}$.

So if Spoiler chooses $\alpha^{\prime}=\alpha-1, \beta^{\prime}=\beta-1$ and moves $p m \stackrel{a}{\longrightarrow} p^{\prime} m^{\prime}$ then any possible response by Duplicator will take the game to a position $\left(p^{\prime} m^{\prime}, q^{\prime} n^{\prime}, \gamma, \alpha^{\prime}\right)$ for a $\gamma \leq \beta$. By induction hypothesis Spoiler wins the game.

Lemma 11 For all ordinals $\alpha, \beta$ the following properties hold.

1. $p m \preceq_{\alpha}^{\beta}$ qn implies $p m^{\prime} \preceq_{\alpha}^{\beta} q n^{\prime}$ for all $m^{\prime} \leq m$ and $n^{\prime} \geq n$

2. If $\alpha^{\prime} \geq \alpha$ and $\beta^{\prime} \geq \beta$ then $\preceq_{\alpha^{\prime}}^{\beta^{\prime}} \subseteq \preceq_{\alpha}^{\beta}$.

3. There are ordinals $C A, C B$ such that $\preceq_{C A}=\preceq_{C A+1}$ and $\preceq^{C B}=\preceq^{C B+1}$.

4. $\preceq=\bigcap_{\alpha} \preceq_{\alpha}=\bigcap_{\beta} \preceq^{\beta}$

The first point states that individual approximants are monotonic in the sense of Proposition 4. Points 2.-4. imply that both $\preceq_{\alpha}$ and $\preceq^{\beta}$ yield non-increasing sequences of approximants that converge towards simulation. It is easy to see that the approximants $\preceq_{\alpha}$ do not converge at finite levels, and not even at $\omega$, i.e., $C A>\omega$ in general. However, we will show that the approximants $\preceq^{\beta}$ do converge at a finite level, i.e., $C B \in \mathbb{N}$ for any pair of nets.

Proof 1) By Lemma 10 it suffices to observe that Duplicator can reuse a winning strategy in the approximant game from $(p m, q n, \alpha, \beta)$ to win the game from $\left(p m-d_{1}, q n+d_{2}, \alpha, \beta\right)$ for naturals $d_{1}, d_{2}$.

2) If $p m \preceq_{\alpha^{\prime}}^{\beta^{\prime}} q n$ then, by Lemma 10, Duplicator wins the approximant game from position $\left(p m, q n, \beta^{\prime}, \alpha^{\prime}\right)$. By Lemma 9 he can also win the approximant game from $(p m, q n, \beta, \alpha)$. Thus pm $\preceq_{\alpha}^{\beta}$ qn by Lemma 10 .

3) By point 2) we see that with increasing ordinal index $\alpha$ the approximant relations $\preceq_{\alpha}$ form a decreasing sequence of relations, thus they stabilize for some ordinal $C A$. The existence of a convergence ordinal for $\preceq^{C B}$ follows analogously.

4) First we observe that $\bigcap_{\alpha} \preceq_{\alpha}=\bigcap_{\alpha} \bigcap_{\beta} \preceq_{\alpha}^{\beta}=\bigcap_{\beta} \bigcap_{\alpha} \preceq_{\alpha}^{\beta}=\bigcap_{\beta} \preceq^{\beta}$. It remains to show that $\preceq=\bigcap_{\alpha} \preceq_{\alpha}$.

To show $\preceq \supseteq \bigcap_{\alpha} \preceq_{\alpha}$, we use $C A$ from point 3 ) and rewrite the right side to $\bigcap_{\alpha} \preceq_{\alpha}=\preceq_{C A}$ $=\preceq_{C A+1}$. From Definition 6 we get that $\preceq_{\alpha}=\preceq_{\alpha}^{\gamma}$ for $\gamma \geq \alpha$ and therefore $\preceq_{C A+1}^{C A+1}=\preceq_{C A+1}=$ 
$\preceq_{C A}=\preceq_{C A}^{C A}$. This means $\preceq_{C A}^{C A}=\bigcap_{\alpha} \preceq_{\alpha}$ must be a simulation relation and hence a subset of $\preceq$.

To show $\preceq \subseteq \bigcap_{\alpha} \preceq_{\alpha}$, we prove by ordinal induction that $\preceq \subseteq \preceq_{\alpha}$ for all ordinals $\alpha$. The base case $\alpha=0$ is trivial. For the induction step we prove the equivalent property $\npreceq \alpha \subseteq \npreceq$. There are two cases.

In the first case, $\alpha=\alpha^{\prime}+1$ is a successor ordinal. If $p m \npreceq \alpha_{\alpha^{\prime}+1} q n$ then $p m \npreceq_{\alpha^{\prime}+1}^{\alpha^{\prime}+1} q n$ and therefore, by Lemma 10, Spoiler wins the approximant game from $\left(p m, q n, \alpha^{\prime}+1, \alpha^{\prime}+1\right)$. Let $p m \stackrel{a}{\longrightarrow} p^{\prime} m^{\prime}$ be an optimal initial move by Spoiler. Now either there is no valid response and thus Spoiler immediately wins in the simulation game or for every Duplicator response $q n \stackrel{a}{\longrightarrow} q^{\prime} n^{\prime}$ we have $p^{\prime} m^{\prime} \npreceq_{\alpha^{\prime}}^{\alpha^{\prime}} q^{\prime} n^{\prime}$. Then also $p^{\prime} m^{\prime} \npreceq_{\alpha^{\prime}} q^{\prime} n^{\prime}$ and by induction hypothesis $p^{\prime} m^{\prime} \npreceq q^{\prime} n^{\prime}$. By Proposition 2 we obtain that Spoiler wins the simulation game from $\left(p^{\prime} m^{\prime}, q^{\prime} n^{\prime}\right)$ and thus from $(p m, q n)$. Therefore $p m \npreceq q n$, as required.

In the second case, $\alpha$ is a limit ordinal. Then $p m \npreceq \swarrow_{\alpha} q n$ implies $p m \npreceq_{\alpha^{\prime}} q n$ for some $\alpha^{\prime}<\alpha$ and therefore $p m \npreceq q n$ by induction hypothesis.

The following lemma shows a certain uniformity property of the simulation game. Beyond some fixed bound, an increased counter value of Spoiler can be neutralized by an increased counter value of Duplicator, thus enabling Duplicator to survive at least as many rounds in the game as before.

Lemma 12 For any one-counter net $N=(Q, A, \delta)$ and $\omega$-net $N^{\prime}=\left(Q^{\prime} A, \delta^{\prime}\right)$ there is a fixed bound $c \in \mathbb{N}$ s.t. for all states $p \in Q, q \in Q^{\prime}$, naturals $m^{\prime}>m>c$ and ordinals $\alpha$ :

$$
\forall n .\left(p m \preceq_{\alpha} q n \Longrightarrow \exists n^{\prime} \cdot p m^{\prime} \preceq_{\alpha} q n^{\prime}\right)
$$

Proof It suffices to show the existence of a local bound $c$ for any given pair of states $p, q$ that satisfies (3), since we can simply take the global $c$ to be the maximal such bound over all finitely many pairs $p, q$. Consider now a fixed pair $p, q$ of states. For $m, n \in \mathbb{N}$, we define the following (sequences of) ordinals.

$$
\begin{aligned}
I(m, n)= & \text { the largest ordinal } \alpha \text { with } p m \preceq_{\alpha} \text { qn or } C A \\
& \text { if no such } \alpha \text { exists, } \\
I(m)= & \text { the increasing sequence of ordinals } I(m, n)_{n \geq 0}, \\
S(m)= & \sup \{I(m)\} .
\end{aligned}
$$

Observe that $I(m, n)$ can be presented as an infinite matrix where $I(m)$ is a column and $S(m)$ is the limit of the sequence of elements of column $I(m)$ looking upwards. Informally, $S(m)=\lim _{i \rightarrow \infty} I(m, i)$.

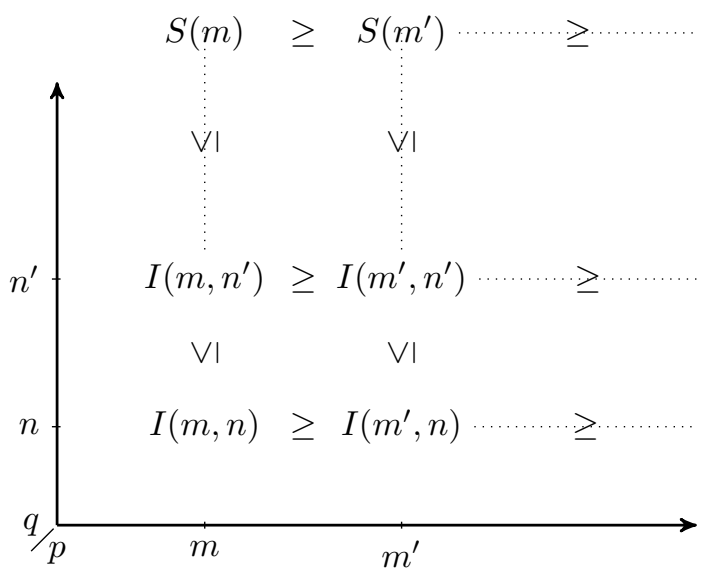


By Lemma 11 (point 1), we derive that for any $n^{\prime}>n \in \mathbb{N}$ and $m^{\prime}>m \in \mathbb{N}$

$$
I\left(m, n^{\prime}\right) \geq I(m, n) \geq I\left(m^{\prime}, n\right)
$$

and because of the second inequality also $S(m) \geq S\left(m^{\prime}\right)$. So the ordinal sequence $S(m)_{m \geq 0}$ of suprema must be non-increasing and by the well-ordering of the ordinals there is a smallest index $k \in \mathbb{N}$ at which this sequence stabilizes:

$$
\forall l>k . S(l)=S(k) .
$$

We split the remainder of this proof into three cases depending on whether $I(k)$ and $I(l)$ for some $l>k$ have maximal elements. In each case we show the existence of a bound $c$ that satisfies requirement (3).

Case 1. For all $l \geq k$ and $n \in \mathbb{N}$ it holds that $I(l, n)<S(l)$, i.e., no $I(l)$ has a maximal element. In this case $c:=k$ satisfies the requirement (3). To see this, take $m^{\prime}>m>c=k$ and $p m \preceq_{\alpha} q n$. Then, by our assumption, $\alpha<S(m)$ and $S(m)=S\left(m^{\prime}\right)=S(k)$. Therefore $\alpha<S\left(m^{\prime}\right)$. Thus there must exist an $n^{\prime} \in \mathbb{N}$ s.t. $p m^{\prime} \preceq_{\alpha} q n^{\prime}$, as required.

Case 2. For all $l \geq k$ there is a $n_{l} \in \mathbb{N}$ such that $I\left(l, n_{l}\right)=S(l)$, i.e., all $I(l)$ have maximal element $S(l)=S(k)$. Again $c:=k$ satisfies the requirement (3). Given $m^{\prime}>m>c=k$ and $p m \preceq_{\alpha} q n$ we let $n^{\prime}:=n_{m^{\prime}}$ and obtain $I\left(m^{\prime}, n^{\prime}\right)=S\left(m^{\prime}\right)=S(k) \geq \alpha$ and thus $p m^{\prime} \preceq_{\alpha} q n^{\prime}$, as required.

Case 3. If none of the two cases above holds then there must exist some $l>k$ s.t. the sequences $I(k), \ldots, I(l-1)$ each have a maximal element and for $l^{\prime}>l$ the sequence $I\left(l^{\prime}\right)$ has no maximal element. To see this, consider sequences $I(x)$ and $I\left(x^{\prime}\right)$ with $x^{\prime}>x \geq k$. If $I\left(x^{\prime}\right)$ has a maximal element then so must $I(x)$, by equation (4) and $S(x)=S\left(x^{\prime}\right)=S(k)$. Given this, we repeat the argument of the Case 1 . with $c:=l$ and again satisfy the requirement (3).

Lemma 13 Consider strong simulation $\preceq$ between a one-counter net $N=(Q, A, \delta)$ and an $\omega$-net $N^{\prime}=\left(Q^{\prime}, A, \delta^{\prime}\right)$. There exists a constant $C B \in \mathbb{N}$ s.t. $\preceq=\preceq C B$.

Proof We assume the contrary and derive a contradiction. By Lemma 11(4), the inclusion $\preceq \subseteq \preceq^{\beta}$ always holds for every ordinal $\beta$. Thus, if $\nexists C B \in \mathbb{N}$. $\preceq=\preceq C B$ then for every finite $\beta \in \mathbb{N}$ there are processes $p_{0} m_{0}$ and $q_{0} n_{0}$ s.t. $p_{0} m_{0} \preceq^{\beta} q_{0} n_{0}$ but $p_{0} m_{0} \npreceq q_{0} n_{0}$. In particular, this holds for the special case of $\beta=\left|Q \times Q^{\prime}\right|(c+1)$, where $c$ is the constant given by Lemma 12, which we consider in the rest of this proof.

Since $q_{0} n_{0}$ does not simulate $p_{0} m_{0}$, we can assume a winning strategy for Spoiler in the simulation game which is optimal in the sense that it guarantees that the simulation level $\alpha_{i}$ - the largest ordinal with $p_{i} m_{i} \preceq_{\alpha_{i}} q_{i} n_{i}$ - strictly decreases along rounds of any play. By monotonicity (Lemma 11, part 1) we can thus infer that whenever a pair of control-states repeats along a play, then Duplicator's counter must have decreased or Spoiler's counter must have increased: Along any partial play

$\left(p_{0} m_{0}, q_{0} n_{0}\right)\left(t_{0}, t_{0}^{\prime}\right)\left(p_{1} m_{1}, q_{1} n_{1}\right)\left(t_{t}, t_{1}^{\prime}\right) \ldots\left(p_{k} m_{k}, q_{k} n_{k}\right)$ of length $k$ with $p_{i}=p_{j}$ and $q_{i}=q_{j}$ for some $i<j \leq k$ we have $n_{j}<n_{i}$ or $m_{j}>m_{i}$. By a similar argument we can assume that Duplicator also plays optimally, in the sense that he uses $\omega$-transitions to increase his counter to higher values than in previous situations with the same pair of control-states. By combining this with the previously stated property that the sequence of $\alpha_{i}$ strictly decreases we obtain the following:

$$
\text { if } p_{i}=p_{j}, q_{i}=q_{j} \text { and } t_{i-1}^{\prime}, t_{j-1}^{\prime} \in \delta_{\omega} \text { then } m_{j}>m_{i} \text {. }
$$

Here $\delta_{\omega}$ denotes the set of transitions with symbolic effect $\omega$ in Duplicator's net. 
Although Duplicator loses the simulation game between $p_{0} m_{0}$ and $q_{0} n_{0}$, our assumption $p_{0} m_{0} \preceq^{\beta} q_{0} n_{0}$ with $\beta=\left|Q \times Q^{\prime}\right|(c+1)$ implies that Duplicator does not lose with less than $\beta \omega$-transitions, regardless of Spoiler's strategy. Thus there always is a prefix of a play along which Duplicator makes use of $\omega$-transitions $\beta$ times.

Let $\pi=\left(p_{0} m_{0}, q_{0} n_{0}\right)\left(t_{0}, t_{0}^{\prime}\right)\left(p_{1} m_{1}, q_{1} n_{1}\right)\left(t_{t}, t_{1}^{\prime}\right) \ldots\left(p_{k} m_{k}, q_{k} n_{k}\right)$ be such a partial play.

Our choice of $\beta=\left|Q \times Q^{\prime}\right|(c+1)$ guarantees that some pair $(p, q)$ of control-states repeats at least $c+1$ times directly after Duplicator making an $\omega$-step. Thus there are indices $i(1)<$ $i(2)<\cdots<i(c+1)<k$ s.t. for all $1 \leq j \leq c+1$ we have $p_{i(j)}=p, q_{i(j)}=q$ and $t_{i(j)}^{\prime} \in \delta_{\omega}$. By observation (5) and $m_{0} \geq 0$ we obtain that $m_{i(x)} \geq x$ for $0 \leq x \leq c+1$. In particular, $c \leq m_{i(c)}<m_{i(c+1)}$, i.e., both of Spoiler's counter values after the last two such repetitions must lie above $c$. This allows us to apply Lemma 12 to derive a contradiction.

Let $\alpha$ be the simulation level before this repetition: $\alpha$ is the largest ordinal with $p m_{i(c)} \preceq_{\alpha}$ $q n_{i(c)}$. Since $m_{i(c+1)}>m_{i(c)}>c$, Lemma 12 ensures the existence of a natural $n^{\prime}$ s.t. $p m_{i(c+1)} \preceq_{\alpha}$ $q n^{\prime}$. Because Duplicator used an $\omega$-transition in his last response leading to the repetition of states there must be a partial play $\pi^{\prime}$ in which both players make the same moves as in $\pi$ except that Duplicator chooses $n_{i(c+1)}$ to be $n^{\prime}$. Now in this play we observe that the simulation level did in fact not strictly decrease as this last repetition of control-states shows: We have $p m_{i(c)} \preceq_{\alpha} q n_{i(c)} \nsucceq_{\alpha+1} p m_{i(c)}$ and $p m_{i(c+1)} \preceq_{\alpha} q n_{i(c+1)}$, which contradicts the optimality of Spoiler's strategy.

\section{The Main Result}

We prove the decidability of simulation between one-counter nets and $\omega$-nets. First, we show that for each finite level $k \in \mathbb{N}$ the approximant $\preceq^{k}$ is effectively semilinear, i.e., we can compute the semilinearity description of $\preceq^{k}$. This yields a decision procedure for simulation that works as follows. Iteratively compute $\preceq^{k}$ for growing $k$ and check after each round if the approximant has converged yet. The convergence test of $\preceq^{k} \stackrel{?}{=} \preceq^{k-1}$ can easily be done, since the approximants are semilinear sets. Termination of this procedure is guaranteed by Lemma 13, and the limit is the simulation relation by Lemma 11 (point 4 ).

We recall the following important result by Jančar, Kučera and Moller.

Theorem 14 ([11]) The largest strong simulation relation $\preceq$ between processes of two given one-counter nets is effectively semilinear.

Now we construct the semilinear approximants $\preceq^{k}$.

Lemma 15 Given a one-counter net $N$ and an $\omega$-net $N^{\prime}$, the approximant relations $\preceq^{k}$ between them are effectively semilinear sets for all $k \in \mathbb{N}$.

Proof Let $N=(Q, A c t, \delta)$ and $N^{\prime}=\left(Q^{\prime}\right.$, Act,$\left.\delta^{\prime}\right)$. We prove the effective semilinearity of $\preceq^{k}$ by induction on $k$.

The base case $\preceq^{0}=Q \times \mathbb{N} \times Q^{\prime} \times \mathbb{N}$ is trivially effectively semilinear.

For the induction step we proceed as follows. By induction hypothesis $\preceq^{k}$ is effectively semilinear. Using this, we reduce the problem of checking $\preceq^{k+1}$ between $N$ and $N^{\prime}$ to the problem of checking normal strong simulation $\preceq$ between two derived one-counter nets $N_{S}$ and $N_{D}$, and obtain the effective semilinearity of the relation from Theorem 14. More precisely, the derived one-counter nets $N_{S}$ and $N_{D}$ will contain all control-states of $N$ and $N^{\prime}$, respectively, and we will have that $p m \preceq^{k+1} q n$ w.r.t. $N, N^{\prime}$ iff $p m \preceq q n$ w.r.t. $N_{S}, N_{D}$.

Before we describe $N_{S}$ and $N_{D}$ formally, we explain the function of a certain test gadget used in the construction. 
An important observation is that after Duplicator made an $\omega$-move in the approximation game between $N$ and $N^{\prime}$, the winner of the game from the resulting configuration depends only on the control-states and Spoiler's counter value, because Duplicator could choose his counter arbitrarily high. Moreover, monotonicity (Lemma 11, point 1) guarantees that there must be a minimal value for Spoiler's counter with which he can win if at all. This yields the following property.

For any pair of states $(p, q) \in Q \times Q^{\prime}$ there must exist a value $M(p, q) \in \mathbb{N} \cup\{\omega\}$ s.t. for all $m \in \mathbb{N}$

$$
\left(\forall n \in \mathbb{N} . p m \swarrow^{k} q n\right) \Longleftrightarrow m \geq M(p, q)
$$

Since, by induction hypothesis, $\preceq^{k}$ (and thus also its complement) is effectively semilinear, we can compute the values $M(p, q)$ for all $(p, q) \in Q \times Q^{\prime}$.

The test gadgets. Given the values $M(p, q)$, we construct test gadgets that check whether Spoiler's counter value is $\geq M(p, q)$. For each $(p, q) \in Q \times Q^{\prime}$ we construct two one-counter nets $S(p, q)$ and $T(p, q)$ with initial states $s(p, q)$ and $t(p, q)$, respectively, such that the following property holds for all $m, n \in \mathbb{N}$.

$$
s(p, q) m \npreceq t(p, q) n \Longleftrightarrow m \geq M(p, q)
$$

The construction of $S(p, q)$ and $T(p, q)$ is very simple. Let $s(p, q)$ be the starting point of a counter-decreasing chain of $e$-steps of length $M(p, q) \in \mathbb{N}$ where the last state of the chain can make an $f$-step whereas $t(p, q)$ is a simple $e$-loop (where $e, f$ are fresh actions not in Act). If $M(p, q)=\omega$, making $s(p, q)$ a deadlock suffices. Thus $S(p, q)$ and $T(p, q)$ are one-counter nets, denoted by $S(p, q)=\left(Q_{s}(p, q), \operatorname{Act}_{s}(p, q), \delta_{s}(p, q)\right)$ and $T(p, q)=\left(Q_{t}(p, q), \operatorname{Act}_{t}(p, q), \delta_{t}(p, q)\right)$. Wlog. we assume that their state sets are disjoint from each other and from the original nets $N, N^{\prime}$.

The construction of $N_{S}$ and $N_{D}$. Let $N_{S}=\left(Q_{S}, A c t^{\prime}, \delta_{S}\right)$ and $N_{D}=\left(Q_{D}, A c t^{\prime}, \delta_{D}\right)$ be one-counter nets constructed as follows. $A c t^{\prime}=A c t \cup Q \times Q^{\prime} \cup\{f, e\}$ (where $e, f$ are the actions from the test gadgets). Spoiler's new net $N_{S}$ has states

$$
Q_{S}=Q \cup \bigcup_{p \in Q, q \in Q^{\prime}} Q_{s}(p, q)
$$

Duplicator's new net $N_{D}$ has states

$$
Q_{D}=Q^{\prime} \cup\{W\} \cup \bigcup_{p \in Q, q \in Q^{\prime}} Q_{t}(p, q) .
$$

where $W$ is a new state.

Now we define the transition relations. $\delta_{S}=\delta \cup \bigcup_{p \in Q, q \in Q^{\prime}} \delta_{s}(p, q)$ plus the following transitions for all $p \in Q, q \in Q^{\prime}$ :

$$
p \stackrel{(p, q), 0}{\longrightarrow} s(p, q)
$$

$\delta_{D}=\left\{q \stackrel{a, x}{\longrightarrow} q^{\prime} \in \delta^{\prime} \mid x \neq \omega\right\} \cup \bigcup_{p \in Q, q \in Q^{\prime}} \delta_{t}(p, q)$ plus the following transitions for all $p, p^{\prime} \in Q$ 
and $q, q^{\prime} \in Q^{\prime}$ :

$$
\begin{aligned}
q \stackrel{a, 0}{\longrightarrow} t\left(p, q^{\prime}\right) & , \quad \text { if } q \stackrel{a, \omega}{\longrightarrow} q^{\prime} \in \delta^{\prime} \\
q \stackrel{\left(p^{\prime}, q^{\prime}\right), 0}{\longrightarrow} W & \\
t(p, q) \stackrel{(p, q), 0}{\longrightarrow} t(p, q) & \\
t(p, q) \stackrel{\left(p, q^{\prime}\right), 0}{\longrightarrow} W & \text { for all } q \neq q^{\prime} \\
t(p, q) \stackrel{a, 0}{\longrightarrow} W & \text { for all } a \in A c t \\
W \stackrel{a, 0}{\longrightarrow} W & \text { for all } a \in A c t^{\prime}
\end{aligned}
$$

Correctness proof. We show that for any pair pm, qn of configurations of the nets $N, N^{\prime}$ we have $p m \preceq^{k+1} q n$ if and only if $p m \preceq q n$ in the newly constructed nets $N_{S}, N_{D}$.

To prove the 'if' direction we assume that $p m \npreceq^{k+1} q n$ w.r.t. $N, N^{\prime}$ and derive that $p m \npreceq q n$ w.r.t. $N_{S}, N_{D}$. By our assumption and Definition 6 , there exists some ordinal $\alpha$ s.t. $p m \npreceq_{\alpha}^{k+1}$ $q n$. By Lemma 10, Spoiler has a winning strategy in the approximation game from position $(p m, q n, \alpha, k+1)$. The result then follows from the following general property.

Property P1. For all ordinals $\alpha$, control-states $(p, q) \in Q \times Q^{\prime}$ and naturals $m, n \in \mathbb{N}$ : If Spoiler has a winning strategy in the approximation game from position $(p m, q n, \alpha, k+1)$ then he also has a winning strategy in the strong simulation game between $N_{S}, N_{D}$ from position $(p m, q n)$.

Proof To prove P1, we fix some $p \in Q, q \in Q^{\prime}$ and proceed by ordinal induction on $\alpha$. The base case trivially holds since Spoiler looses from a position $(p m, q n, 0, k+1)$.

For the induction step let Spoiler play the same move $p m \stackrel{a}{\longrightarrow} p^{\prime} m^{\prime}$ for some $a \in$ Act in both games according to his assumed winning strategy in the approximation game. Now Duplicator makes his response move in the new game between $N_{S}, N_{D}$, which yields two cases. In the first case, Duplicator does not use a transition from Equation (11). Then his move induces a corresponding move in the approximation game which leads to a new configuration $\left(p^{\prime} m^{\prime}, q^{\prime} n^{\prime}, \alpha^{\prime}, k+1\right)$ where $p^{\prime} m^{\prime} \npreceq_{\alpha^{\prime}}^{k+1} q^{\prime} n^{\prime}$ for some ordinal $\alpha^{\prime}<\alpha$. Thus, by Lemma 10 and the induction hypothesis, the property holds.

In the second case, Duplicator's response is via a transition from Equation (11), which leads to a new configuration $\left(p^{\prime} m^{\prime}, t\left(p^{\prime \prime}, q^{\prime}\right) n\right)$ for some $p^{\prime \prime} \in Q$. Thus in the approximants game there will exist Duplicator moves to positions $\left(p^{\prime} m^{\prime}, q^{\prime} n^{\prime}, \alpha^{\prime}, k\right)$ where $n^{\prime} \in \mathbb{N}$ can be arbitrarily high. We can safely assume that Duplicator chooses $p^{\prime \prime}=p^{\prime}$, since otherwise Spoiler can win in one round by playing $p^{\prime} m^{\prime} \stackrel{\left(p^{\prime}, q^{\prime}\right)}{\longrightarrow}$. Now in the following round Spoiler can play $p^{\prime} m^{\prime} \stackrel{\left(p^{\prime}, q^{\prime}\right)}{\longrightarrow} s\left(p^{\prime}, q^{\prime}\right) m^{\prime}$ by Equation (10) and Duplicator's only option is to stay in his current state by Equation (13). The game thus continues from $\left(s\left(p^{\prime}, q^{\prime}\right) m^{\prime}, t\left(p^{\prime}, q^{\prime}\right) n\right)$. By our assumption Spoiler wins the approximation game from the position $(p m, q n, \alpha, k+1)$. Thus there is some ordinal $\alpha^{\prime}<\alpha$ s.t. Spoiler also wins the approximation game from the position $\left(p^{\prime} m^{\prime}, q^{\prime} n^{\prime}, \alpha^{\prime}, k\right)$ for every $n^{\prime} \in \mathbb{N}$. Thus, by Lemma 10 and Definition 6, we have $p^{\prime} m^{\prime} \swarrow_{\alpha^{\prime}}^{k} q^{\prime} n^{\prime}$ and by Lemma 11 (item 2) $p^{\prime} m^{\prime} \swarrow^{k} q^{\prime} n^{\prime}$ for all $n^{\prime} \in \mathbb{N}$. By Equation (6) we obtain $m^{\prime} \geq M\left(p^{\prime}, q^{\prime}\right)$. By the construction of the gadgets and Equation (7) we get $s\left(p^{\prime}, q^{\prime}\right) m^{\prime} \npreceq t\left(p^{\prime}, q^{\prime}\right) n$, which implies the desired property. $\square$ This concludes the proof of the 'if' direction. Now we prove the 'only if' direction of the correctness property. We assume that $p m \npreceq q n$ in the newly constructed nets $N_{S}$ and $N_{D}$ and derive that $p m \npreceq^{k+1} q n$ w.r.t. $N, N^{\prime}$. To do this, we first show the following general property. 
Property P2. If $p m \npreceq q n$ with respect to nets $N_{S}$ and $N_{D}$ then there exists some general ordinal $\alpha^{\prime}$ s.t. $p m \npreceq_{\alpha^{\prime}}^{k+1} q n$ with respect to nets $N, N^{\prime}$. Proof Assume $p m \npreceq q n$ with respect to nets $N_{S}$ and $N_{D}$. Since both $N_{S}, N_{D}$ are just one-counter nets, non-simulation manifests itself at some finite approximant $\alpha \in \mathbb{N}$, i.e., $p m \npreceq_{\alpha} q n$. We prove property P2 by induction on $\alpha$. The base case of $\alpha=0$ is trivial. For the induction step we consider a move $p m \stackrel{a}{\longrightarrow} p^{\prime} m^{\prime}$ for some $a \in A c t$ by Spoiler in both games according to Spoiler's assumed winning strategy in the game between $N_{S}, N_{D}$ (It cannot be a Spoiler move $p \stackrel{(p, q), 0}{\longrightarrow} s(p, q)$ by Equation (10), because Duplicator would immediately win via a reply move by Equation (12)). Now we consider all (possibly infinitely many) replies by Duplicator in the approximation game between $N, N^{\prime}$ from a position $\left(p m, q n, \alpha^{\prime}, k+1\right)$ for some yet to be determined ordinal $\alpha^{\prime}$. These replies fall into two classes.

In the first class, Duplicator's move $q n \stackrel{a}{\longrightarrow} q^{\prime} n^{\prime}$ is not due to an $\omega$-transition and thus also a possible move in the strong simulation game between $N_{S}, N_{D}$. From our assumption that Spoiler wins the strong simulation game from position $(p m, q n)$ in at most $\alpha$ steps, it follows that Spoiler wins the strong simulation game from $\left(p^{\prime} m^{\prime}, q^{\prime} n^{\prime}\right)$ in at most $\alpha-1$ steps. By induction hypothesis, there exists an ordinal $\alpha^{\prime \prime}$ s.t. Spoiler has a winning strategy in the approximation game for $\preceq_{\alpha^{\prime \prime}}^{k+1}$ between $N, N^{\prime}$ from position $\left(p^{\prime} m^{\prime}, q^{\prime} n^{\prime}\right)$. There are only finitely many such replies. Thus let $\alpha^{0}$ be the maximal such $\alpha^{\prime \prime}$.

In the second class, Duplicator's move $q n \stackrel{a}{\longrightarrow} q^{\prime} n^{\prime}$ uses an $\omega$-transition which does not exist in $N_{D}$. Instead there exists a Duplicator transition $q n \stackrel{a, 0}{\longrightarrow} t\left(p^{\prime \prime}, q^{\prime}\right) n$ by Equation (11). From our assumption that Spoiler wins the strong simulation game from position $(p m, q n)$ in at most $\alpha$ steps, it follows that Spoiler wins the strong simulation game from $\left(p^{\prime} m^{\prime}, t\left(p^{\prime \prime}, q^{\prime}\right) n\right)$ in at most $\alpha-1$ steps. If $p^{\prime \prime} \neq p^{\prime}$ then this is trivially true by a Spoiler move by Equation (10). Otherwise, if $p^{\prime \prime}=p^{\prime}$, then this can only be achieved by a Spoiler move of $p^{\prime} m^{\prime} \stackrel{\left(p^{\prime}, q^{\prime}\right), 0}{\longrightarrow} s\left(p^{\prime}, q^{\prime}\right) m^{\prime}$ in the next round, because for any other Spoiler move Duplicator has a winning countermove by Equations (14) or (15). In this case Duplicator can only reply with a move $t\left(p^{\prime}, q^{\prime}\right) q n \stackrel{\left(p^{\prime}, q^{\prime}\right), 0}{\longrightarrow} t\left(p^{\prime}, q^{\prime}\right) n$ by Equation (13), and we must have that Spoiler can win in at most $\alpha-2$ steps from position $\left(s\left(p^{\prime}, q^{\prime}\right) m^{\prime}, t\left(p^{\prime}, q^{\prime}\right) n\right)$. This implies, by Equation (7), that $m^{\prime} \geq M\left(p^{\prime}, q^{\prime}\right)$. Then Equation (6) yields $\forall n \in \mathbb{N}$. $p^{\prime} m^{\prime} \npreceq^{k} q^{\prime} n$. Thus for every $n \in \mathbb{N}$ there exists some ordinal $\alpha_{n}$ s.t. $p^{\prime} m^{\prime} \npreceq_{\alpha_{n}}^{k} q^{\prime} n$. Let $\alpha^{\prime \prime}$ be the smallest ordinal s.t. $\forall n \in \mathbb{N} . \alpha_{n} \leq \alpha^{\prime \prime}$. Each of the finitely many distinct $\omega$-transitions yields such an $\alpha^{\prime \prime}$. Let $\alpha^{1}$ be the maximum of them.

We set $\alpha^{\prime}:=\max \left(\alpha^{0}, \alpha^{1}\right)+1$. Then every reply to Spoilers move $p m \stackrel{a}{\longrightarrow} p^{\prime} m^{\prime}$ in the approximation game from $\left(p m, q n, \alpha^{\prime}, k+1\right)$ leads to some position that is winning for Spoiler. So, Spoiler has a winning strategy in the approximation game from $\left(p m, q n, \alpha^{\prime}, k+1\right)$ and by Lemma 10, pm $\preceq_{\alpha^{\prime}}^{k+1} q n$ w.r.t. $N, N^{\prime}$, which concludes the proof of property P2. $\quad \square$ To show the 'only if' direction of the correctness property, we assume that $p m \npreceq q n$ in the newly constructed nets $N_{S}, N_{D}$. By property P2 we have $p m \npreceq_{\alpha^{\prime}}^{k+1} q n$ for some ordinal $\alpha^{\prime}$ and thus $p m \swarrow^{k+1} q n$ w.r.t. $N, N^{\prime}$. This concludes the 'only if' direction.

We have constructed one-counter nets $N_{S}, N_{D}$ s.t. $p m \preceq^{k+1} q n$ w.r.t. $N, N^{\prime}$ if and only if $p m \preceq q n$ w.r.t. $N_{S}, N_{D}$. By Theorem $14, \preceq^{k+1}$ is effectively semilinear.

Theorem 16 The largest weak simulation over processes of a given one-counter net is effectively semilinear and thus decidable.

Proof By Theorem 5 it suffices to show that the largest strong simulation relation $\preceq$ between a one-counter net $N$ and a $\omega$-net $N^{\prime}$ is effectively semilinear. By Lemma 15 , we can iteratively compute the semilinearity description of the approximants $\preceq^{k}$ for $k=0,1,2, \ldots$. Convergence can be detected by checking if $\preceq^{k} \stackrel{?}{=} \preceq^{k-1}$, which is effective because equality is decidable for 
semilinear sets. Termination (i.e., eventual convergence at a finite index) of this procedure is guaranteed by Lemma 13, and the reached limit is the semilinear simulation relation by Lemma 11 (item 4).

\section{Approximant Convergence at $\omega^{2}$}

We show that ordinary weak simulation approximants $\leqq_{\alpha}$ converge at level $\alpha=\omega^{2}$ on OCN.

Lemma 17 When considering relations between a one-counter net and an $\omega$-net, we have $\preceq_{\omega i}$ $\subseteq \preceq^{i}$ for every $i \in \mathbb{N}$.

Proof By induction on $i$. The base case of $i=0$ is trivial, since $\preceq^{0}$ is the full relation. We prove the inductive step by assuming the contrary and deriving a contradiction. Let $p m \preceq_{\omega i} q n$ and $p m \npreceq^{i} q n$ for some $i>0$. Then there exists some ordinal $\alpha$ s.t. $p m \npreceq_{\alpha}^{i} q n$. Without restriction let $\alpha$ be the least ordinal satisfying this condition. If $\alpha \leq \omega i$ then we trivially have a contradiction. Now we consider the case $\alpha>\omega i$. By $p m \npreceq_{\alpha}^{i} q n$ and Lemma 10, Spoiler has a winning strategy in the approximant game from position $(p m, q n, \alpha, i)$. Without restriction we assume that Spoiler plays optimally, i.e., wins as quickly as possible. Thus this game must reach some game position $\left(p^{\prime} m^{\prime}, q^{\prime} n^{\prime}, \alpha^{\prime}+1, i\right)$ where $\alpha^{\prime} \geq \omega i$ is a limit ordinal, such that Spoiler can win from $\left(p^{\prime} m^{\prime}, q^{\prime} n^{\prime}, \alpha^{\prime}+1, i\right)$ but not from $\left(p^{\prime} m^{\prime}, q^{\prime} n^{\prime}, \alpha^{\prime}, i\right)$. I.e., $p^{\prime} m^{\prime} \swarrow_{\alpha^{\prime}+1}^{i} q^{\prime} n^{\prime}$, but $p^{\prime} m^{\prime} \preceq_{\alpha^{\prime}}^{i} q^{\prime} n^{\prime}$. Consider Spoiler's move $p^{\prime} m^{\prime} \stackrel{a}{\longrightarrow} p^{\prime \prime} m^{\prime \prime}$ according to his optimal winning strategy in the game from position $\left(p^{\prime} m^{\prime}, q^{\prime} n^{\prime}, \alpha^{\prime}+1, i\right)$. Since $p^{\prime} m^{\prime} \preceq_{\alpha^{\prime}}^{i} q^{\prime} n^{\prime}$ and $\alpha^{\prime}$ is a limit ordinal, for every ordinal $\gamma_{k}<\alpha^{\prime}$, Duplicator must have some countermove $q^{\prime} n^{\prime} \stackrel{a}{\longrightarrow} q_{k} n_{k}$ s.t. $p^{\prime \prime} m^{\prime \prime} \preceq_{\gamma_{k}}^{j} q_{k} n_{k}$, where $j=i-1$ if the move was due to an $\omega$-transition and $j=i$ otherwise. In particular, $\sup _{k}\left\{\gamma_{k}\right\}=\alpha^{\prime}$. However, since Spoiler's move $p^{\prime} m^{\prime} \stackrel{a}{\longrightarrow} p^{\prime \prime} m^{\prime \prime}$ was according to his optimal winning strategy from position $\left(p^{\prime} m^{\prime}, q^{\prime} n^{\prime}, \alpha^{\prime}+1, i\right)$, we have that $p^{\prime \prime} m^{\prime \prime} \npreceq_{\alpha^{\prime}}^{j} q_{k} n_{k}$. Therefore, there must be infinitely many different Duplicator countermoves $q^{\prime} n^{\prime} \stackrel{a}{\longrightarrow} q_{k} n_{k}$. Infinitely many of these countermoves must be due to an $\omega$-transition, because apart from these the system is finitely branching. Thus for every ordinal $\gamma<\alpha^{\prime}$ there is some Duplicator countermove $q^{\prime} n^{\prime} \stackrel{a}{\longrightarrow} q_{k} n_{k}$ which is due to an $\omega$-transition s.t. $p^{\prime \prime} m^{\prime \prime} \preceq_{\gamma_{k}}^{i-1} q_{k} n_{k}$ where $\gamma_{k} \geq \gamma$ (note the $i-1$ index due to the $\omega$-transition). In particular, we can choose $\gamma=\omega(i-1)$, because $i>0$ and $\alpha^{\prime} \geq \omega i$. Then we have $p^{\prime \prime} m^{\prime \prime} \preceq_{\omega(i-1)}^{i-1} q_{k} n_{k}$, but $p^{\prime \prime} m^{\prime \prime} \swarrow_{\alpha^{\prime}}^{i-1} q_{k} n_{k}$. However, from $p^{\prime \prime} m^{\prime \prime} \preceq_{\omega(i-1)}^{i-1} q_{k} n_{k}$ and the induction hypothesis, we obtain $p^{\prime \prime} m^{\prime \prime} \preceq^{i-1} q_{k} n_{k}$ and in particular $p^{\prime \prime} m^{\prime \prime} \preceq_{\alpha^{\prime}}^{i-1} q_{k} n_{k}$. Contradiction.

Theorem 18 Weak simulation approximants on OCN converge at level $\omega^{2}$, but not earlier in general.

Proof First we show that $\leqq_{\omega^{2}}$ is contained in $\leqq$ for OCN. Let $p m$ and $q n$ be processes of OCN $M$ and $N$, respectively. Let $M^{\prime}, N^{\prime}$ be the derived OCN and $\omega$-net from Theorem 5 . Assume $p m \leqq_{\omega^{2}} q n$ w.r.t. $M, N$. Then, by point 2) of Theorem $5, p m \preceq_{\omega^{2}} q n$ w.r.t. $M^{\prime}, N^{\prime}$. In particular we have $p m \preceq_{\omega C B} q n$ w.r.t. $M^{\prime}, N^{\prime}$, with the $C B \in \mathbb{N}$ from Lemma 13. From Lemma 17 we obtain $p m \preceq \preceq^{C B} q n$ w.r.t. $M^{\prime}, N^{\prime}$. Lemma 13 yields $p m \preceq q n$ w.r.t. $M^{\prime}, N^{\prime}$. Finally, by Theorem 5 , we obtain $p m \leqq q n$ w.r.t. $M, N$.

To see that $\omega^{2}$ is needed in general, consider the following class of examples. Let $p \stackrel{a}{\longrightarrow} p$ define a simple OCN (actually even a finite system). For every $i \in \mathbb{N}$ we define an OCN $N_{i}$ with transitions $\left(q_{k}, a,-1, q_{k}\right),\left(q_{k-1}, \tau, 0, q_{k-1}^{\prime}\right),\left(q_{k-1}^{\prime}, \tau, 1, q_{k-1}^{\prime}\right)$, and $\left(q_{k-1}^{\prime}, a, 0, q_{k}\right)$ for all $k$ with $1 \leq k \leq i$. Then, for the net $N_{i}$, we have $p \leqq_{\omega i} q_{0} 0$, but $p \not q_{0} 0$. Thus in general $\leqq_{\omega i}$ for any $i \in \mathbb{N}$. 


\section{Undecidability of Trace Inclusion and Equivalence}

For any process $\alpha$ we write $T(\alpha)$ for the set $\left\{w \in A c t^{*} \mid \exists \beta . \alpha \stackrel{w}{\longrightarrow} \beta\right\}$ of traces of $\alpha$. We consider trace inclusion and equivalence checking for OCN, which was stated as an open question in [4]. We show that both problems are undecidable for OCN by a reduction from the containment problem for weighted automata [2].

Definition 19 (Weighted Automata) $A$ weighted finite automaton (WFA) is a tuple $\left(Q, \Sigma, \delta, q_{0}\right)$ where $Q$ is a finite set of states, $\Sigma$ a finite alphabet, $q_{0} \in Q$ an initial state and $\delta \subseteq Q \times \Sigma \times \mathbb{N} \times Q$ a transition relation. If $\left(p, a, d, p^{\prime}\right) \in \delta$ the automaton can go from $p$ to $p^{\prime}$ reading a symbol ' $a$ ' with reward $d \in \mathbb{N}$. A run of $A$ on a word $w=w_{0} w_{1} \ldots w_{n} \in \Sigma^{*}$ is a sequence $\left(q_{i}, w_{i}, d_{i}, q_{i+1}\right)_{0 \leq i<n} \in \delta^{*}$ of transitions. The value of such a run is $\sum_{i=0}^{n-1} d_{i}$; the value $L(A, w)$ of a word $w \in \Sigma^{*}$ is the maximal value of any run on $w$.

We say that the language of WFA $A$ is contained in that of WFA $B$ (over the same alphabet $\Sigma), L(A) \subseteq L(B)$, iff for all words $w \in \Sigma^{*}, L(A, w) \leq L(B, w)$. Checking $L(A) \subseteq L(B)$ is undecidable [2] (Theorem 4$)$. The next result is a direct consequence.

Theorem 20 Trace inclusion/equivalence between OCN processes is undecidable.

Proof Inclusion can trivially be reduced to equivalence for nondeterministic systems like OCN. Thus we show undecidability of inclusion by reduction from WFA containment. The idea is to encode the WFA as OCN, using the counter as accumulator. To ensure a faithful encoding of WFA containment, the OCN can at any point jump to a gadget that compares the counter values.

Given WFA $A=\left(Q_{A}, \Sigma, \delta_{A}, q_{A}\right)$ and $B=\left(Q_{B}, \Sigma, \delta_{B}, q_{B}\right)$ we construct nets $A^{\prime}$ and $B^{\prime}$ with states $Q_{A} \cup\{D\}$ and $Q_{B} \cup\{D\}$ resp., over alphabet $A c t=\Sigma \cup\{d\}$ where $d$ is a fresh symbol. We add transitions $D \stackrel{d,-1}{\longrightarrow} D$ to both nets as well as $q \stackrel{d,-1}{\longrightarrow} D$ for any original state. We argue that $L(A) \subseteq L(B)$ if and only if $T\left(q_{A}(0)\right) \subseteq T\left(q_{B}(0)\right)$.

Assume a witness $w$ with $L(A, w)=v>L(B, w)$. Then there is a run of $A$ on $w$ with a value higher than that of any run of $B$ on $w$. So the word $w d^{v}$ must be a valid trace from $q_{A}(0)$, but not from $q_{B}(0)$. Conversely, if $L(A, w) \leq L(B, w)$ for all $w \in \Sigma^{*}$, then for any run of $A^{\prime}$ there is a run of $B^{\prime}$ over the same sequence of actions which accumulates a higher or equal counter value. Thus no such word can be extended to a counterexample for trace inclusion by appending finitely many $d$ 's.

\section{Comparing OCN/OCA and Finite Systems}

Simulation. First we consider checking strong/weak simulation between OCN/OCA and finite systems, and vice-versa.

Theorem 21 Checking if a finite-state process weakly simulates a OCN process is in $P$.

Proof It suffices to first replace the step relation in the finite system with its weak closure so that $q \stackrel{a}{\Longrightarrow} q^{\prime} \Longleftrightarrow q \stackrel{a}{\longrightarrow} q^{\prime}$ and then check if the resulting finite system strongly simulates the net. The finiteness of the state space allows us to compute the weak closure in polynomial time. A polynomial time algorithm for checking strong simulation between OCN and finite-state processes can be found in [16].

For the other direction, checking if a OCN process weakly simulates a finite-state process, we show that it suffices to consider a finite version of the net where the counter is capped 
at a polynomially bounded level. The crucial observation is that monotonicity implies that Duplicator must be able to ensure that his counter never decreases along any partial play that repeats control-states.

Definition 22 Let $N=(Q, A c t, \delta)$ be a $O C N$ and $l \in \mathbb{N}$. The $l$-capped version of $N$ is the finite system $N_{l}=\left(Q_{l}, \longrightarrow\right)$ with states $Q_{l}=\{(\hat{q}, n) \mid q \in Q, n \leq l\}$ and transitions $(\hat{q}, n) \stackrel{a}{\longrightarrow}\left(\hat{q}^{\prime}, \min \left\{n^{\prime}, l\right\}\right)$ iff $q n \stackrel{a}{\longrightarrow} q_{N} n^{\prime}$.

It is easy to see that $N_{l}$ can be constructed from $N$ in time proportional to $l \times|N|$. For $n, l \in \mathbb{N}$ we observe the following properties.

\section{Proposition 23}

1. $(\hat{q}, \min \{n, l\}) \leqq q n$,

2. $q n \leqq_{l}(\hat{q}, \min \{n, l\})$,

3. $(\hat{q}, \min \{n, l\}) \leqq(\hat{q}, \min \{n+1, l\})$.

We continue to show that Duplicator can be assumed to play optimally in a sense that depends on cycles in the underlying control graphs. Consider a simulation game between a finite process and a OCN process (or its $c$-capped version).

Definition 24 A partial play $\pi=\left(p_{0}, q_{0} n_{0}\right)\left(t_{0}, t_{0}^{\prime}\right) \ldots\left(p_{l}, q_{l} n_{l}\right)$ is a cycle if $p_{0}=p_{l}$ and $q_{0}=q_{l}$. It is decreasing if $n_{0}>n_{l}$.

Similarly, if Duplicator plays on a c-capped version of the net, the play $\pi=\left(p_{0},\left(\hat{q}_{0}, \min \left\{n_{0}, c\right\}\right)\right)$ $\left(t_{0}, t_{0}^{\prime}\right) \ldots\left(p_{l},\left(\hat{q}_{l}, \min \left\{n_{l}, c\right\}\right)\right)$ is a cycle if $p_{0}=p_{l}$ and $\hat{q}_{0}=\hat{q}_{i}$ and is decreasing if the second component of Duplicator's state decreases. A cycle is called simple if no proper subsequence is itself a cycle. The length of simple cycles is bounded by $|S \times Q|$, where $S$ is the set of states of the finite-state process.

Lemma 25 Suppose $p \leqq q n$. Then Duplicator has a winning strategy in the weak simulation game that moreover guarantees the following properties in every play.

1. No round decreases the counter by more than $|Q| * 2+1$.

2. Every simple cycle is non-decreasing.

Proof A weak step $s_{0}\left(m_{0}\right) \stackrel{a}{\Longrightarrow} t_{l}\left(n_{l}\right)$ by Duplicator is due to some sequence $s_{0}\left(m_{0}\right) \stackrel{\tau}{\longrightarrow} s_{1}\left(m_{1}\right)$ $\stackrel{\tau}{\longrightarrow} \ldots \stackrel{\tau}{\longrightarrow} s_{l}\left(m_{l}\right) \stackrel{a}{\longrightarrow} t_{0}\left(n_{0}\right) \stackrel{\tau}{\longrightarrow} t_{1}\left(n_{1}\right) \stackrel{\tau}{\longrightarrow} \ldots \stackrel{\tau}{\longrightarrow} t_{k}\left(n_{k}\right)$. By monotonicity it is suboptimal for Duplicator to decrease the counter when silently moving from state $s_{i}$ to $s_{j}=s_{i}$ (or from $t_{i}$ to $t_{j}=t_{i}$ ) for $i<j$. Also, we can safely assume that a weak step as above will be non-decreasing if there are indices $i<j$ with $s_{i}=s_{j}$ and $m_{i}<m_{j}$ (or $t_{i}=t_{j}$ and $n_{i}<n_{j}$ ). Therefore, if the weak step decreases the counter, both silent paths will be acyclic and hence no longer than $|Q|$. Such a step cannot decrease the counter by more than $|Q| * 2+1$.

For the second point observe that if Duplicator cannot avoid the next simple cycle to be decreasing, then Spoiler must have some strategy to enforce cycles to be decreasing. Such a strategy must be winning for Spoiler as it eventually exhausts Duplicator's counter.

The next lemma uses the previously stated optimality assumption to show that we only need to consider a polynomially capped net to determine if a OCN process weakly simulates a finite-state process. 
Lemma 26 For any pair $F=(S, \longrightarrow), N=(Q$, Act,$\delta)$ of a finite-state system and OCN resp., there is a fixed polynomial bound $c$ such that for all $n \in \mathbb{N}$ :

$$
p \leqq q n \Longleftrightarrow p \leqq(\hat{q}, \min \{n, c\})
$$

and $(\hat{q}, \min \{n, c\})$ is a state of the c-capped version $N_{c}$ of $N$.

Proof The "if" direction follows directly from Proposition 23 (point 1). For the other direction we show that $c=(2|Q|+1)(|S \times Q|+1)$ suffices to contradict $p \leqq q n$ and $p \geq(\hat{q}, \min \{n, c\})$.

If $p \leqq q n$ then $p \leqq_{c} q n$ and by Proposition 23 (2) we have $p \leqq_{c}(\hat{q}, \min \{n, c\})$. Moreover, Duplicator has an optimal strategy in the sense of Lemma 25 . We see that using the same strategy in the game $p$ vs. $(\hat{q}, \min \{n, c\})$ guarantees that

1. No round decreases the second component of Duplicator's state by more than $|Q| * 2+1$.

2. For any simple cycle between game positions $p_{i},\left(\hat{q}_{i}, n_{i}\right)$ and $p_{j},\left(\hat{q}_{j}, n_{j}\right)$ it holds that $n_{j} \geq n_{i}$ or $n_{j} \geq c-(|Q| * 2+1) *(|S \times Q|)$.

To see the second point observe that the only way a simple cycle can be decreasing is because some of its increases are dropped due to the counter being at its limit $c$. Then point 1 implies $n_{j} \geq c-(2|Q|+1)(|S \times Q|)$ because the length of simple cycles is bounded by $|S \times Q|$.

By our assumption $p \not z(\hat{q}, \min \{n, c\})$, we can consider a play

$$
\pi=\left(p_{0},\left(\hat{q}_{0}, n_{0}\right)\right)\left(t_{0}, t_{0}^{\prime}\right)\left(p_{1},\left(\hat{q}_{1}, n_{1}\right)\right)\left(t_{1}, t_{1}^{\prime}\right) \ldots\left(p_{l},\left(\hat{q}_{l}, n_{l}\right)\right)
$$

where $p_{0}=p$ and $\left(\hat{q}_{0}, n_{0}\right)=(\hat{q}, \min \{n, c\})$, along which Duplicator plays optimally as described above and which is winning for Spoiler in the smallest possible number of rounds.

Since $c>|S \times Q|$, we know that $\pi$ must contain cycles as otherwise $l \leq|S \times Q|$ and thus $p \leqq_{c}(q, \min \{n, c\})$ contradicts that $\pi$ is won by Spoiler. So assume the last simple cycle in $\pi$ is between positions $i$ and $j$. We know that $n_{j}<n_{i}$, as otherwise omitting this last cycle results in a shorter winning play for Spoiler by monotonicity.

Thus, by Observation 2 above, $n_{j} \geq c-(2|Q|+1)(|S \times Q|)$ and in particular we get that $n_{j} \geq(2|Q|+1)$ due to our choice of $c$.

Finally, recall that the last position $p_{l},\left(\hat{q}_{l}, n_{l}\right)$ in the play $\pi$ must be directly winning for Spoiler. That is, for some action $a$ it holds that $p_{l} \stackrel{a}{\longrightarrow}$ and $\left(\hat{q}_{l}, n_{l}\right) \stackrel{a}{\Rightarrow}$. But since $n_{l}>(2|Q|+1)$, we know that also $q_{l} n_{l} \nRightarrow$ in the original OCN process. This contradicts our assumption that Duplicator's original strategy in the unrestricted game was winning.

Theorem 27 Checking if a OCN process weakly simulates a finite-state process can be done in polynomial time.

Proof To check if $p \leqq q n$ holds we can by Lemma 26 equivalently check $p \leqq(\hat{q}, \min \{n, c\})$ where $(\hat{q}, \min \{n, c\})$ is a state of a polynomially bounded finite system $N_{c}$. Checking weak simulation between two finite processes is in $P$.

Trace Inclusion. Now we consider checking strong/weak trace inclusion between finite-state systems and OCA/OCN. It is undecidable whether an OCA contains the strong/weak traces of a finite-state system [22]. However, is is decidable whether a Petri net contains the strong/weak traces of a finite-state system [10], and thus the question is decidable for OCN.

Now we consider the other direction of trace inclusion. We show that checking whether a finite-state system contains the strong/weak traces of an OCA is PSPACE complete. For this we recall some structural properties of OCA processes. We write $p m \longrightarrow_{k} q n$ for OCA configurations 
$p m$ and $q n$ if there is a path of length $k$ from $p m$ to $q n$. A path is positive if at most the last visited configuration has counter value 0 , i.e., no step is due to a transition in $\delta_{0}$. We write $p m \stackrel{+}{\longrightarrow} q n$ if there is a positive path of length $k$ from $p m$ to $q n$.

Lemma 28 ([5], Lemma 5) Consider a OCA with stateset $Q$ and let $p, q \in Q$. If $p 1 \longrightarrow^{*} q 0$ then $p 1 \longrightarrow_{k} q 0$ for some $k \leq|Q|^{3}$.

Lemma 29 Consider a OCA $\left(Q, A c t, \delta, \delta_{0}\right)$ where $K=|Q|$ and $p, q \in Q$. If $p m \longrightarrow^{*} q n$ for some $n \in \mathbb{N}$ then $p m \longrightarrow q q n^{\prime}$ for some $n^{\prime}$ and $k \leq \max \{m, 1\} 5 K^{4}$.

Proof We distinguish two cases depending on whether there is a positive minimal path from $p m$ to $q$.

Case 1: There is a positive minimal path witnessing $p m \longrightarrow^{*} q n$. Consider such a path $\left(p_{0} m_{0}\right),\left(p_{1} m_{1}\right), \ldots,\left(p_{k} m_{k}\right)$ from $p m=p_{0} m_{0}$ to $q n=p_{k} m_{k}$. We know that there is a path from $p$ to $q$ in the control graph of the automaton that uses transitions in $\delta$ only. So there must be such a path in the control graph that is no longer than $K$. Thus, if $m \geq K$, then there is a $n^{\prime}$ such that $p m \stackrel{+}{\longrightarrow} q n^{\prime}$ for some $k \leq K$. Otherwise, if $m<K$, we observe that

$$
\text { if } p m \stackrel{+}{\rightarrow} p^{\prime} m^{\prime} \text { then } p(m+1) \stackrel{+}{\longrightarrow} p^{\prime}\left(m^{\prime}+1\right) \text {. }
$$

After at most $K$ steps, our minimal path will repeat some control-state $p_{j}=p_{l}$ at positions $j<l<K$. By minimality and point (17) we can assume that $m_{j}<m_{l}$. Therefore, after at most $K$ such repetitions the counter will reach a value $\geq K$, and thus, by the first case above, the remaining path must be of length $\leq K$. This allows us to bound the length of the minimal path from $p m$ to control-state $q$ by $K^{2}+K$.

Case 2: No minimal path witnessing $p m \longrightarrow^{*} q n$ is positive. Consider a minimal path $\left(p_{0} m_{0}\right),\left(p_{1} m_{1}\right), \ldots,\left(p_{k} m_{k}\right)$ from $p m=p_{0} m_{0}$ to $q n=p_{k} m_{k}$ and let $i_{0}, i_{1}, \ldots, i_{l}$ be exactly those indices with $m_{i_{j}}=0$. We split the path into phases $p_{0} m_{0} \longrightarrow{ }^{*} p_{i_{0}} m_{i_{0}}, p_{i_{j}} m_{i_{j}+1} \longrightarrow{ }^{*} p_{i_{(j+1)}} m_{\left.i_{(j+1}\right)}$ for $0 \leq j<l$ and $p_{i_{(l+1)}} m_{i_{(l+1)}} \longrightarrow^{*} p_{k} m_{k}$ and consider the first, the last and the intermediate phases separately.

First phase. The path $p_{0} m_{0} \longrightarrow{ }^{*} p_{i_{0}} m_{i_{0}}$ can be split into parts $q_{j}\left(m_{0}-j\right) \longrightarrow^{*} q_{j+1}\left(m_{0}-\right.$ $(j+1))$ for $0 \leq j<m_{0}$ and $q_{0}=p_{0}$ and $q_{m_{0}}=p_{i_{0}}$, by considering the first occasions where the counter value reaches $m_{0}-j$. In particular, inside the path $q_{j}\left(m_{0}-j\right) \longrightarrow^{*} q_{j+1}\left(m_{0}-(j+1)\right)$ the counter value does not drop below $m_{0}-j$ before the last step. By Lemma 28 and point (17) there exists a path $q_{j}\left(m_{0}-j\right) \longrightarrow^{*} q_{j+1}\left(m_{0}-(j+1)\right)$ of length $\leq K^{3}$. Thus the path $p_{0} m_{0} \longrightarrow^{*} p_{i_{0}} m_{i_{0}}$ can be bounded by length $m K^{3}$.

Intermediate phases. These are paths from some configuration $p_{i_{j}} 0$ to $p_{i_{(j+1)}} 0$. Such a path is either of length 1 , or the first step increases the counter, i.e., $p_{i_{j}} 0 \rightarrow q 1$ for some control-state $q$. In the latter case, by minimality and Lemma 28 we can bound the path from $q 1$ to $p_{i_{(j+1)}} 0$ by $K^{3}$. Thus we can bound the path $p_{i_{j}} 0$ to $p_{i_{(j+1)}} 0$ by $K^{3}+1$. Note that there can only be at most $K$ such intermediate phases, because the path would otherwise repeat a configuration which would contradict its minimality.

Last phase. The last phase is a positive path. Like in Case 1) we can bound its length by $K^{2}+K$.

To conclude, the length of the shortest witness for $p m \longrightarrow^{*} q n$ is bounded by $m K^{3}+K\left(K^{3}+\right.$ 1) $+K^{2}+K \leq \max \{m, 1\} 5 K^{4}$.

Theorem 30 Checking strong trace inclusion $T(p m) \subseteq T(q)$ or weak trace inclusion $T(p m) \subseteq$ $T(q)$ for a OCA process $p m$ and a finite process $q$ is PSPACE complete. 
Table 1: Decidability of preorders and equivalences on finite-state systems, OCN and OCA, resp. New results in boldface.

\begin{tabular}{|l|c|c|c|}
\hline & FS & OCN & OCA \\
\hline$\sim$ & P-complete [19] & \multicolumn{2}{|c|}{ PSPACE-complete [21,3] } \\
\hline$\approx$ & P-complete [19] & \multicolumn{2}{|c|}{ undecidable [17] } \\
\hline$\preceq$ & P-complete [19] & $\begin{array}{c}\text { decidable [1, 11] } \\
\text { PSPACE-hard [21] }\end{array}$ & undecidable [13] \\
\hline$\leqq$ & P-complete [19] & decidable & undecidable [13] \\
\hline$\subseteq / \subseteq$ & PSPACE-compl. [18] & undecidable & undecidable [22] \\
\hline
\end{tabular}

Table 2: Known results on checking simulation, weak simulation and trace inclusion between one-counter and finite systems.

\begin{tabular}{|l|c|c|}
\hline & OCN & OCA \\
\hline$\sim F S$ & P-complete [15] & P-complete [15] \\
\hline$\approx F S$ & $P^{N P}$-complete [8] & $P^{N P}$-complete [8] \\
\hline$\preceq F S$ (and $F S \preceq)$ & P-complete [16] & PSPACE-complete[20, 21] \\
\hline$\leqq F S($ and $F S \leqq)$ & P-complete & PSPACE-complete[20, 21] \\
\hline$\subseteq / \subseteq F S$ & PSPACE-complete & PSPACE-complete \\
\hline$F S \subseteq / \subseteq$ & decidable [10] & undecidable [22] \\
\hline
\end{tabular}

Proof A PSPACE lower bound holds already for strong trace inclusion of finite-state systems [18]. The weak trace inclusion problem $T(p m) \subseteq T(q)$ can trivially be reduced to the strong one by taking the transitive closure of the finite system w.r.t. invisible transitions. It remains to show a PSPACE upper bound for the problem $T(p m) \subseteq T(q)$. Let $p m$ be a configuration of the OCA $A=\left(Q, A c t, \delta, \delta_{0}\right)$ and $q$ a state of the NFA $B=(S, A c t, \delta)$ and let $\bar{B}$ denote the powerset automaton of $B$.

To check if $T(p m) \nsubseteq \nsubseteq T(q)$ holds we can equivalently test $T(p m) \cap T(q)^{c} \neq \emptyset$. That is, if in the product automaton $A \times \bar{B}$ some control-state $\left(p^{\prime}, \emptyset\right)$ is reachable from initial configuration $(p,\{q\}) m$. This can be checked by nondeterministically guessing a path stepwise. The finite control of the automaton $A \times \bar{B}$ is bounded by $K:=|Q| * 2^{|S|}$. By Lemma 29 we know that the shortest path that witnesses such a control-state reachability is bounded by $B:=\max \{m, 1\} 5 K^{4}$. This bounds the number of steps we need to consider until we can safely terminate and conclude that in fact trace inclusion holds. $B$ is polynomial in $m$ and $|Q|$ and exponential in $|S|$. However, we need only polynomial space to store a configuration of $A \times \bar{B}$ (with control-state numbers and counter values encoded in binary) and the binary coded values of the search-depth and its bound $B$. Thus we can check the condition in PSPACE.

\section{Summary and Conclusion}

We summarize known results about the complexity of checking the following semantic preorders/equivalences: strong bisimulation $\sim$, weak bisimulation $\approx$, strong simulation $\preceq$, weak simulation $\leqq$, strong trace inclusion $\subseteq$ and weak trace inclusion $\subseteq$. In Table 1 we consider problems where systems of the same type are compared, while in Table 2 we consider the problems of checking preorders/equivalences between infinite-state systems and finite-state systems.

The construction used to show PSPACE hardness of strong bisimulation in [21] uses OCN only, and moreover it can be modified to prove a PSPACE lower bound for checking strong simulation between OCA and finite systems (and vice-versa) and strong simulation for OCN; 
see Remark 3.8 in [21].

The proof of the undecidability of weak bisimulation between OCN [17] can be modified to work even for the subclass of normed nets with unary alphabets.

A PSPACE upper bound for strong/weak simulation between OCA and FS (and vice-versa) can be obtained by reduction to $\mu$-calculus model checking for OCA, which is in PSPACE [20].

\section{References}

[1] P. A. Abdulla and K. Cerans. Simulation is decidable for one-counter nets (extended abstract). In CONCUR, volume 1466 of LNCS, 1998.

[2] S. Almagor, U. Boker, and O. Kupferman. What's decidable about weighted automata? In ATVA, volume 6996 of LNCS, 2011.

[3] S. Böhm, S. Göller, and P. Jančar. Bisimilarity of one-counter processes is PSPACEcomplete. In CONCUR, volume 6269 of LNCS, 2010.

[4] J. Esparza and M. Nielsen. Decibility issues for Petri nets - a survey. Journal of Informatik Processing and Cybernetics, 30(3):143-160, 1994.

[5] K. Etessami, D. Wojtczak, and M. Yannakakis. Quasi-birth-death processes, tree-like QBDs, probabilistic 1-counter automata, and pushdown systems. In QEST, pages 243-253. IEEE, 2008 .

[6] R. v. Glabbeek. The linear time - branching time spectrum I; the semantics of concrete, sequential processes. In J. Bergstra, A. Ponse, and S. Smolka, editors, Handbook of Process Algebra, chapter 1, pages 3-99. Elsevier, 2001.

[7] S. Göller and M. Lohrey. Branching-time model checking of one-counter processes. In STACS, volume 5 of LIPIcs, pages 405-416. Schloss Dagstuhl - Leibniz-Zentrum fuer Informatik, 2010.

[8] S. Göller, R. Mayr, and A. W. To. On the computational complexity of verifying one-counter processes. In LICS, pages 235-244. IEEE, 2009.

[9] P. Jančar. Undecidability of bisimilarity for Petri nets and some related problems. Theor. Comput. Sci., 148(2):281-301, 1995.

[10] P. Jančar, J. Esparza, and F. Moller. Petri nets and regular processes. J. Comput. Syst. Sci., 59(3):476-503, 1999.

[11] P. Jančar, A. Kučera, and F. Moller. Simulation and bisimulation over one-counter processes. In STACS, volume 1770 of $L N C S, 2000$.

[12] P. Jančar and F. Moller. Simulation of one-counter nets via colouring. Technical report, Uppsala Computing Science, February 1999.

[13] P. Jančar, F. Moller, and Z. Sawa. Simulation problems for one-counter machines. In SOFSEM, volume 1725 of LNCS, pages 404-413, 1999.

[14] A. Kučera and R. Mayr. Why is simulation harder than bisimulation? In CONCUR, volume 2421 of LNCS, pages 594-609. Springer, 2002. 
[15] A. Kučera. Efficient verification algorithms for one-counter processes. In ICALP, volume 1853 of $L N C S$, pages 317-328, 2000.

[16] A. Kučera. On simulation-checking with sequential systems. In ASIAN, volume 1961 of LNCS, pages 133-148, 2000.

[17] R. Mayr. Undecidability of weak bisimulation equivalence for 1-counter processes. In ICALP, volume 2719 of $L N C S$, pages 570-583, 2003.

[18] A. Meyer and L. Stockmeyer. The equivalence problem for regular expressions with squaring requires exponential space. In Symp. Foundations of Computer Science, pages 125-129. IEEE, 1972.

[19] Z. Sawa and P. Jančar. P-hardness of equivalence testing on finite-state processes. In SOFSEM, volume 2234 of LNCS, 2001.

[20] O. Serre. Parity games played on transition graphs of one-counter processes. In FoSSaCS, volume 3921 of $L N C S$, pages 337-351, 2006.

[21] J. Srba. Beyond language equivalence on visibly pushdown automata. Logical Methods in Computer Science, 5(1):1-22, 2009.

[22] L. Valiant. Decision procedures for families of deterministic pushdown automata. PhD thesis, Department of Computer Science, University of Warwick, Coventry, July 1973. 


\section{A Proof of Theorem 5}

Theorem 5. For two one-counter nets $M$ and $N$ with states $Q_{M}$ and $Q_{N}$ resp., one can effectively construct a OCN $M^{\prime}$ with states $Q_{M^{\prime}} \supseteq Q_{M}$ and a $\omega$-net $N^{\prime}$ with states $Q_{N^{\prime}} \supseteq Q_{N}$ such that for each pair $p, q \in Q_{M} \times Q_{N}$ of original control states and any ordinal $\alpha$ the following hold.

1. $p m \leqq q n$ w.r.t. $M, N$ iff $p m \preceq q n$ w.r.t. $M^{\prime}, N^{\prime}$.

2. If $p m \leqq_{\alpha} q n$ w.r.t. $M, N$ then $p m \preceq_{\alpha} q n$ w.r.t. $M^{\prime}, N^{\prime}$.

The reduction will be done in two steps. First (Lemma 35) we reduce weak simulation for one-counter nets to strong simulation beteween a one-counter net and yet another auxiliary model called guarded $\omega$-nets. These differ from $\omega$-nets in that each transition may change the counter by more than one and is guarded by an integer, i.e. can only be applied if the current counter value exceeds the guard attached to it. In the second step (Lemma 36) we normalize the effects of all transitions to $\{-1,0,1, \omega\}$ and eliminate all integer guards and thereby construct an ordinary $\omega$-net for Duplicator.

Before we start observe that without loss of generality we can assume that every state $p$ allows a silent loop $p \stackrel{\varepsilon, 0}{\longrightarrow} p$.

Definition $31 A$ path in a one-counter net $N=(Q$, Act,$\delta)$ is a sequence $\pi=\left(s_{0}, a_{0}, d_{0}, t_{0}\right)$ $\left(s_{1}, a_{1}, d_{1}, t_{1}\right) \ldots\left(s_{k}, a_{k}, d_{k}, t_{k}\right) \in \delta^{*}$ of transitions where $s_{i+1}=t_{i}$ for all $i<k$. We call $\pi$ cyclic if $s_{i}=t_{j}$ for some $0 \leq i<j \leq k$ and write ${ }^{i} \pi$ for its prefix of length $i$. A cyclic path is a loop if $p_{i} \neq p_{j}$ for all $0 \leq i<j<k$. Define the effect $\Delta(\pi)$ and guard $\Gamma(\pi)$ of a path $\pi$ by

$$
\Delta(\pi)=\sum_{i=0}^{k} d_{i} \quad \text { and } \quad \Gamma(\pi)=-\min \left\{\Delta\left({ }^{i} \pi\right) \mid i \leq k\right\}
$$

where $n<\omega$ and $n+\omega=\omega+n=\omega$ for every $n \in \mathbb{N}$. The guard $\Gamma(\pi)$ denotes the minimal counter value that is needed to traverse the path $\pi$ while maintaining a non-negative counter value along all intermediate configurations. Lastly, fix a homomorphism obs: $\delta^{*} \rightarrow(\text { Act } \backslash\{\tau\})^{*}$, that maps paths to their observable action sequences: obs $((s, \tau, d, t))=\varepsilon$ and obs $((s, a, d, t))=a$ for $a \neq \tau$.

Definition 32 (Guarded $\omega$-Nets) $A$ guarded $\omega$-net $N=(Q, A c t, \delta)$ is given by finite sets $Q$, Act of states and actions and a transition relation $\delta \subseteq Q \times A c t \times \mathbb{N} \times \mathbb{Z} \cup\{\omega\} \times Q$. It defines a transition system over the stateset $Q \times \mathbb{N}$ where $p m \stackrel{a}{\longrightarrow}$ qn iff there is a transition $(p, a, g, d, q) \in \delta$ with

1. $m \geq g$ and

2. $n=m+d \in \mathbb{N}$ or $d=\omega$ and $n>m$.

Specifically, $N$ is a $\omega$-net if for all transitions $g=0$ and $d \in\{-1,0,1, \omega\}$. The next construction establishes the connection between weak similarity of one-counter nets and strong similarity between OCN and guarded $\omega$-net processes.

Lemma 33 For a one-counter net $N=(Q$, Act,$\delta)$ we can effectively construct a guarded $\omega$-net $G=\left(Q\right.$, Act,$\left.\delta^{\prime}\right)$ such that for all $a \in$ Act,

1. whenever $p m \stackrel{a}{\Longrightarrow}$ qn, there is a $n^{\prime} \geq n$ such that $p m \stackrel{a}{\longrightarrow} q n^{\prime}$ 
2. whenever $p m \stackrel{a}{\longrightarrow} q$, there is a $n^{\prime} \geq n$ such that $p m \stackrel{a}{\Longrightarrow}{ }_{N} q n^{\prime}$.

Proof The idea of the proof is to introduce direct transitions from one state to another for any path between them that reads at most one visible action and does not contain silent cycles.

For two states $s, t$ of $N$, let $D(s, t)$ be the set of direct paths from $s$ to $t$ :

$$
\begin{aligned}
D(s, t)=\{ & \left(p_{i}, a_{i}, d_{i}, p_{i+1}\right)_{i<k}: p_{0}=s, p_{k}=t, \\
& \left.\forall_{0 \leq i<j \leq k} p_{i}=p_{j} \Longrightarrow(i=0 \wedge j=k)\right\} .
\end{aligned}
$$

Define the subset of silent direct paths by $S D(s, t)=\{\pi \in D(s, t) \mid o b s(\pi)=\varepsilon\}$. Every path in $D(s, t)$ has acyclic prefixes only and is therefore bounded in length by $|Q|$. Hence $D(s, t)$ and $S D(s, t)$ are finite and effectively computable for all pairs $s, t$.

Using this notation, we define the transitions in $G$ as follows. Let $\delta^{\prime}$ contain a transition $(p, a, \Gamma(\pi), \Delta(\pi), q)$ for each path $\pi=\pi_{1}\left(s, a, d, s^{\prime}\right) \pi_{2} \in \delta^{+}$where $\pi_{1} \in S D(p, s)$ and $\pi_{2} \in$ $S D\left(s^{\prime}, q\right)$. This carries over all transitions of $N$ because the empty path is in $S D(s, s)$ for all states $s$. Moreover, introduce $\omega$-transitions in case $N$ allows paths $\pi_{1}, \pi_{2}$ as above to contain direct cycles with positive effect on the counter: If there is a path $\pi=\pi_{1}^{\prime} \pi_{1}^{\prime \prime} \pi_{1}^{\prime \prime \prime}\left(s, a, d, s^{\prime}\right) \pi_{2}$ with

1. $\pi_{1}^{\prime} \in S D(p, t), \pi_{1}^{\prime \prime} \in S D(t, t)$ and $\pi_{1}^{\prime \prime \prime} \in S D(t, s)$

2. $\Delta\left(\pi_{1}^{\prime \prime}\right)>0$

for some $t \in Q$, then $\delta^{\prime}$ contains a transition $\left(p, a, \Gamma\left(\pi_{1}^{\prime} \pi_{1}^{\prime \prime}\right), \omega, q\right)$. Similarly, if for some $t \in Q$, there is a path $\pi=\pi_{1}\left(s, a, d, s^{\prime}\right) \pi_{2}^{\prime} \pi_{2}^{\prime \prime} \pi_{2}^{\prime \prime \prime}$ that satisfies

1. $\pi_{1} \in S D(p, s), \pi_{2}^{\prime} \in S D\left(s^{\prime}, t\right), \pi_{2}^{\prime \prime} \in S D(t, t)$ and $\pi_{2}^{\prime \prime \prime} \in S D(t, q)$

2. $\Delta\left(\pi_{2}^{\prime \prime}\right)>0$

add a transition $(p, a, g, \omega, q)$ with guard $g=\Gamma\left(\pi_{1}\left(s, a, d, s^{\prime}\right) \pi_{2}^{\prime} \pi_{2}^{\prime \prime}\right)$. If there is an $a$-labelled path from $p$ to $q$ that contains a silent and direct cycle with positive effect, $G$ has an a-labelled $\omega$-transition from $p$ to $q$ with the guard derived from that path.

To prove the first part of the claim, assume $p m \stackrel{a}{\Longrightarrow}{ }_{N} q n$. By definition of weak steps, there must be a path $\pi=\pi_{1}\left(s, a, d, s^{\prime}\right) \pi_{2}$ with $o b s\left(\pi_{1}\right)=o b s\left(\pi_{2}\right)=\varepsilon$. Suppose both $\pi_{1}$ and $\pi_{2}$ do not contain loops with positive effect. Then there must be paths $\pi_{1}^{\prime} \in S D(p, s), \pi_{2}^{\prime} \in S D\left(s^{\prime}, q\right)$ with $\Gamma\left(\pi_{i}^{\prime}\right) \leq \Gamma\left(\pi_{i}\right)$ and $\Delta\left(\pi_{i}^{\prime}\right) \geq \Delta\left(\pi_{i}\right)$ for $i \in\{1,2\}$ that can be obtained from $\pi_{1}$ and $\pi_{2}$ by removing all loops with effects less or equal 0 . So $G$ contains a transition $\left(p, a, g^{\prime}, d^{\prime}, q\right)$ for some $g^{\prime} \leq m$ and $d^{\prime} \geq n-m$ and hence $p m \stackrel{a}{\longrightarrow} q n^{\prime}$ for $n^{\prime}=m+d^{\prime} \geq n$. Alternatively, either $\pi_{1}$ or $\pi_{2}$ contains a loop with positive effect. Note that for any such path, another path with lower or equal guard exists that connects the same states and contains only one counter-increasing loop: If $\pi_{1}$ contains a loop with positive effect, there is a path $\bar{\pi}_{1}=\pi_{1}^{\prime} \pi_{1}^{\prime \prime} \pi_{1}^{\prime \prime \prime}$ from $p$ to $s$, where $\pi_{1}^{\prime}, \pi^{\prime \prime}$ and $\pi_{1}^{\prime \prime \prime}$ are direct and $\Delta\left(\pi_{1}^{\prime \prime}\right)>0$ for the loop $\pi_{1}^{\prime \prime} \in S D(t, t)$ for some state $t$. In this case, $G$ contains a $\omega$-transition $(p, a, g, \omega, q)$ with $g=\Gamma\left(\pi_{1}^{\prime} \pi_{1}^{\prime \prime}\right)$. Similarly, if $\pi_{2}$ contains the counterincreasing loop, there is a $\overline{\pi_{2}}=\pi_{2}^{\prime} \pi_{2}^{\prime \prime} \pi_{2}^{\prime \prime \prime}$, with $\pi_{2}^{\prime} \in S D\left(s^{\prime}, t\right), \pi_{2}^{\prime \prime} \in S D(t, t), \pi_{2}^{\prime \prime \prime} \in S D(t, q)$ and $\Delta\left(\pi_{2}^{\prime \prime}\right)>0$. This means there is a transition $(p, a, g, \omega, q)$ in $G$ with $g=\Gamma\left(\pi_{1}\left(s, a, d, s^{\prime}\right) \pi_{2}^{\prime} \pi_{2}^{\prime \prime}\right)$. In both cases, $g \leq \Gamma(\pi) \leq m$ and therefore $p m \stackrel{a}{\longrightarrow} q i$ for all $i \geq m$.

For the second part of the claim, assume $p m \stackrel{a}{\longrightarrow} q$ q. This must be the result of a transition $(p, a, g, d, q) \in \delta^{\prime}$ for some $g \leq m$. In case $d \neq \omega$, there is a path $\pi \in \delta^{*}$ from $p$ to $q$ with $\Delta(\pi)=n-m, o b s(\pi)=a$ and $\Gamma(\pi)=g$ that witnesses the weak step $p m \stackrel{a}{\Longrightarrow} q n$ in $N$. Otherwise if $d=\omega$, there must be a path $\pi=\pi_{11} \pi_{12} \pi_{13}\left(s, a, d, s^{\prime}\right) \pi_{21} \pi_{22} \pi_{23}$ from $p$ to $q$ in $N$ where $\Gamma(\pi) \leq m$, all $\pi_{i j}$ are silent and direct and one of $\pi_{12}$ and $\pi_{22}$ is a cycle with strictly positive effect. This implies that one can "pump" the value of the counter higher than any given value. 
Specifically, there are naturals $k$ and $j$ such that the path $\pi^{\prime}=\pi_{11} \pi_{12}^{k} \pi_{13}\left(s, a, d, s^{\prime}\right) \pi_{21} \pi_{22}^{j} \pi_{23}$ from $p$ to $q$ satisfies $\Gamma\left(\pi^{\prime}\right) \leq \Gamma(\pi) \leq m$ and $\Delta\left(\pi^{\prime}\right) \geq m-n$. Now $\pi^{\prime}$ witnesses the weak step $p m \stackrel{a}{\Longrightarrow}{ }_{N} q n^{\prime}$ in $N$ for an $n^{\prime} \geq n$.

Remark 34 Observe that no transition of the net $G$ as constructed above has a guard larger than $|Q| * 3+1$ and finite effect $>2|Q|+1$.

Lemma 35 For a one-counter net $N=(Q$, Act,$\delta)$ one can effectively construct a guarded $\omega$-net $G=\left(Q, A c t, \delta^{\prime}\right)$ s.t. for any $O C N M$ and any two configurations pm, qn of $M$ and $N$ resp.,

$$
p m \leqq q n \text { w.r.t. } M, N \Longleftrightarrow p m \preceq q n \text { w.r.t. } M, G \text {. }
$$

Proof Consider the construction from the proof of Lemma 33. Let $\leqq_{M, N}$ be the largest weak simulation w.r.t. $M, N$ and $\preceq_{M, G}$ be the largest strong simulation w.r.t. $M, G$.

For the "if" direction we show that $\preceq_{M, G}$ is a weak simulation w.r.t. $M, N$. Assume $p m \preceq_{M, G}$ $q n$ and $p m \stackrel{a}{\longrightarrow} p^{\prime} m^{\prime}$. That means there is a step $q n \stackrel{a}{\longrightarrow}_{G} q^{\prime} n^{\prime}$ for some $n^{\prime} \in N$ so that $p^{\prime} m^{\prime} \preceq_{G}$ $q^{\prime} n^{\prime}$. By Lemma 33 part $2, q n \stackrel{a}{\Longrightarrow} q^{\prime} n^{\prime \prime}$ for a $n^{\prime \prime} \geq n^{\prime}$. Because simulation is monotonic we know that also $p^{\prime} m^{\prime} \preceq_{M, G} q^{\prime} n^{\prime \prime}$. Similarly, for the "only if" direction, one can use the first claim of Lemma 33 to check that $\leqq_{M, N}$ is a strong simulation w.r.t. $M, G$.

Lemma 36 For a one-counter net $M$ and a guarded $\omega$-net $G$ one can effectively construct onecounter nets $M^{\prime}, G^{\prime}$ such that for any two configurations pm, qn of $M$ and $G$ resp.,

$$
p m \preceq q n \text { w.r.t. } M, G \Longleftrightarrow p m \preceq q n \text { w.r.t. } M^{\prime}, G^{\prime} .
$$

Proof We first observe that for any transition of the guarded $\omega$-net $G$, the values of its guard is bounded by some constant. The same holds for all finite effects. Let $\Gamma(G)$ be the maximal guard and $\Delta(G)$ be the maximal absolute finite effect of any transition of $G$.

The idea of this construction is to simulate one round of the game $M$ vs. $G$ in $k=2 \Gamma(G)+$ $\Delta(G)+1$ rounds of a simulation game $M^{\prime}$ vs. $G^{\prime}$. We will replace original steps of both players by sequences of $k$ steps in the new game, which is long enough to verify if the guard of Duplicator's move is satisfied and adjust the counter using transitions with effects in $\{-1,0,+1, \omega\}$ only.

We transform the net $M=\left(Q_{M}, A c t, \delta_{M}\right)$ to the net $M^{\prime}=\left(Q_{M^{\prime}}, A c t^{\prime}, \delta_{M^{\prime}}\right)$ as follows:

$$
\begin{aligned}
A c t^{\prime}= & A c t \cup\{b\} \\
Q_{M^{\prime}}= & Q_{M} \cup\left\{p_{i} \mid 1 \leq i<k, p \in Q_{M}\right\} \\
\delta_{M^{\prime}}= & \left\{p \stackrel{a, d}{\longrightarrow} q_{k} \mid p \stackrel{a, d}{\longrightarrow} q \in \delta_{M}\right\} \\
& \cup\left\{p_{i} \stackrel{b, 0}{\longrightarrow} p_{i-1} \mid 1<i<k\right\} \\
& \cup\left\{p_{1} \stackrel{b, 0}{\longrightarrow} q\right\} .
\end{aligned}
$$

We see that

$$
p m \stackrel{a}{\longrightarrow}_{M} q n^{\prime} \Longleftrightarrow p m \stackrel{a}{\longrightarrow}_{M^{\prime}} q_{k-1} n^{\prime b^{k-2}}{\stackrel{\longrightarrow}{M^{\prime}}}_{1} n^{\prime} \stackrel{b}{\longrightarrow}_{M^{\prime}} q n^{\prime} .
$$

Now we transform the guarded $\omega$-net $G=\left(Q_{G}, A c t, \delta_{G}\right)$ to the $\omega$-net $G^{\prime}=\left(Q_{G^{\prime}}, A c t^{\prime}, \delta_{G^{\prime}}\right)$. Every original transition will be replaced by a sequence of $k$ steps that test if the current counter value exceeds the guard $g$ and adjust the counter accordingly. The new net $G^{\prime}$ has states

$$
Q_{G^{\prime}}=Q_{G} \cup\left\{t_{i} \mid 0 \leq i<k, t \in \delta_{G}\right\} .
$$


For each original transition $t=(p, a, g, d, q) \in \delta_{G}$, we add the following transitions to $\delta_{G^{\prime}}$. First, to test the guard:

$$
\begin{aligned}
& p \stackrel{a, 0}{\longrightarrow} t_{k-1}, \\
& t_{i} \stackrel{b,-1}{\longrightarrow} t_{i-1}, \text { for } k-g<i<k \\
& t_{i} \stackrel{b,+1}{\longrightarrow} t_{i-1}, \text { for } k-2 g<i<k-g .
\end{aligned}
$$

Now we add transitions to adjust the counter according to $d \in \mathbb{N} \cup\{\omega\}$. In case $0 \leq d<\omega$ we add

$$
\begin{aligned}
& t_{i} \stackrel{b,+1}{\longrightarrow} t_{i-1}, \text { for } k-2 g-|d|<i<k-2 g \\
& t_{i} \stackrel{b, 0}{\longrightarrow} t_{i-1}, \text { for } 0 \leq i<k-2 g-d .
\end{aligned}
$$

In case $d<0$ we add

$$
\begin{aligned}
& t_{i} \stackrel{b,-1}{\longrightarrow} t_{i-1}, \text { for } k-2 g-|d|<i<k-2 g \\
& t_{i} \stackrel{b, 0}{\longrightarrow} t_{i-1}, \text { for } 0 \leq i<k-2 g+d .
\end{aligned}
$$

In case $d=\omega$ we add

$$
\begin{aligned}
& t_{i} \stackrel{b, \omega}{\longrightarrow} t_{i-1}, \text { for } i=k-2 g \\
& t_{i} \stackrel{b, 0}{\longrightarrow} t_{i-1}, \text { for } 0 \leq i<k-2 g .
\end{aligned}
$$

Finally, we allow a move to the new state:

$$
t_{0} \stackrel{b, 0}{\longrightarrow} q .
$$

Observe that every transition in the constructed net $G^{\prime}$ has effect in $\{-1,0,+1, \omega\} . \quad G^{\prime}$ is therefore an ordinary $\omega$-net. It is streightforward to see that

$$
p m \stackrel{a}{\longrightarrow} q n^{\prime} \Longleftrightarrow p m \stackrel{a b k-1}{\longrightarrow}_{G^{\prime}} q n^{\prime} .
$$

The claim (19) now follows from Equations (25) and (37). This conludes the proof of Lemma 36 and point 1 of Theorem 5 .

For point 2 of the claim observe that by construction of $M^{\prime}$ and $N^{\prime}$, one round of a weak simulation game w.r.t. $M, N$ is simulated by $k$ rounds of a simulation game w.r.t. $M^{\prime}, N^{\prime}$. Therefore, if if Spoiler has a strategy to win the simulation game w.r.t. $M^{\prime}, N^{\prime}$ in $\alpha$ rounds then he can derive a strategy to win the game w.r.t. $M, N$ in not more than $\alpha$ rounds. So if $p m \npreceq_{\alpha} q n$ w.r.t. $M^{\prime}, N^{\prime}$ then $p m \unrhd_{\alpha} q n$ w.r.t. $M, N$. 